\title{
Deformation Quantization on the Closure of Minimal Coadjoint Orbits
}

\author{
CHRISTIAN FRØNSDAL \\ Department of Physics and Astronomy, University of California Los Angeles, Los Angeles, \\ CA 90095-1547, USA. e-mail: fronsdal@physics.ucla.edu \\ Edited with CLAUDE ROGER and FRÉDÉRIC BUTIN \\ Institut Camille Jordan, Université Lyon 1, CNRS UMR5208, 43 blvd du 11 novembre \\ 1918,69622 Villeurbanne-Cedex, France.e-mail: roger@math.univ-lyon1.fr; \\ butin@math.univ-lyon1.fr
}

Received: 31 December 2008 / Revised: 21 March 2009 / Accepted: 21 March 2009

Published online: 21 April 2009 - (C) The Author(s) 2009. This article is published with open access at Springerlink.com

\begin{abstract}
We consider a complex simple Lie algebra $\mathfrak{g}$, with the action of its adjoint group. Among the three canonical nilpotent orbits under this action, the minimal orbit is the non zero orbit of smallest dimension. We are interested in equivariant deformation quantization: we construct $\mathfrak{g}$-invariant star-products on the minimal orbit and on its closure, a singular algebraic variety. We shall make use of Hochschild homology and cohomology, of some results about the invariants of the classical groups, and of some interesting representations of simple Lie algebras. To the minimal orbit is associated a unique, completely prime two-sided ideal of the universal enveloping algebra $U(\mathfrak{g})$. This ideal is primitive and is called the Joseph ideal. We give explicit expressions for the generators of the Joseph ideal and compute the infinitesimal characters.
\end{abstract}

Mathematics Subject Classification (2000). 53D55， 13D03， 17B20， 17B25， $17 \mathrm{~B} 35$.

Keywords. Joseph ideals, Hochschild cohomology, Hochschild homology, minimal coadjoint orbits, quantization, star-products.

\section{Contents}

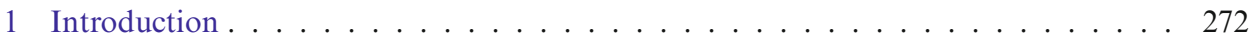

1.1 General framework . . . . . . . . . . . . . . . . . . 272

1.2 History of star-products . . . . . . . . . . . . . . . . . . 273

1.3 Terminology . . . . . . . . . . . . . . . . . . . . . 274

1.4 Deformations and Hochschild cohomology . . . . . . . . . . . . 275

1.5 Results of the article . . . . . . . . . . . . . . . . . 275

1.6 Some recent related works . . . . . . . . . . . . . . . . . 276

1.7 Outline of the paper . . . . . . . . . . . . . . . . . 277

2 Star-products and Hochschild (co)homology . . . . . . . . . . . . . . 278

2.1 Hochschild (co)homology and deformations . . . . . . . . . . . . 278

2.2 The BGS decomposition of Hochschild (co)homology . . . . . . . . . . . 281

2.3 Application of the BGS decomposition to star-products . . . . . . . . 283 
3 Hochschild (co)homology of some varieties with singularities . . . . . . . . . . 284

3.1 Case of quadratic relations: the conic varieties . . . . . . . . . . . . . 284

3.2 Case of one quadratic relation: the simple cone . . . . . . . . . . . 285

3.3 Case of one polynomial relation . . . . . . . . . . . . . 288

4 Introduction to $\mathfrak{g}$-invariant star-products on coadjoint orbits . . . . . . . . . . 289

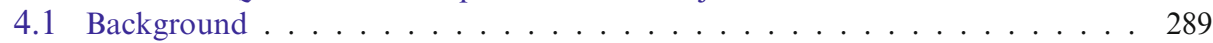

4.2 Definition of $\mathfrak{g}$-invariant star-products . . . . . . . . . . . . . . 289

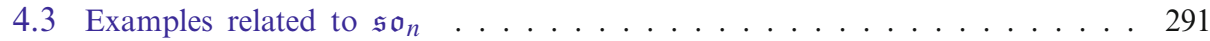

5 Minimal orbits, representations, and Joseph ideals . . . . . . . . . . . . . 292

5.1 Adjoint and coadjoint orbits . . . . . . . . . . . . . . . . . . . . . . . . . . . . . . 292

5.2 Nilpotent orbits and Joseph ideals . . . . . . . . . . . . . . . . 294

5.3 Coadjoint orbits of $\mathfrak{s o}(2,1) \ldots \ldots \ldots \ldots \ldots \ldots$

5.4 Minimal orbit of $\mathfrak{s l}_{n} \ldots \ldots \ldots \ldots \ldots \ldots$

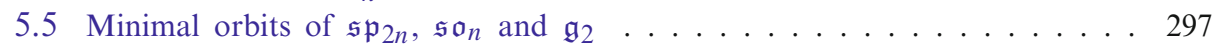

5.6 Associated representations and Joseph ideals . . . . . . . . . . . . . . . . 298

5.6.1. Background . . . . . . . . . . . . . . . . . . . . . . . 298

5.6.2. Associated representations of $\mathfrak{s l}_{n} \ldots \ldots \ldots 299$

5.6.3. Associated representations of $\mathfrak{s o}_{n}$ and $\mathfrak{s p}_{2 n} \ldots \ldots \ldots 300$

$6 \mathfrak{g}$-Invariant star-products on the minimal orbit of a simple Lie algebra . . . . . . 301

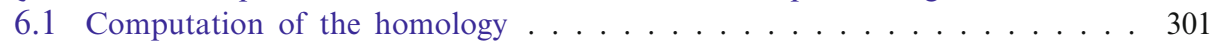

6.2 Correspondence principle for $\mathfrak{g}$-invariant star-products . . . . . . . . . . 302

6.3 Calculations for $\mathfrak{s l}_{n}$ : Joseph ideal and highest weight modules . . . . . . 304

6.3.1. Solving the constraints . . . . . . . . . . . . . . . . . 304

6.3.2. Generators of the Joseph ideal . . . . . . . . . . . . . . . . . . 305

6.3.3. Highest weight module . . . . . . . . . . . . . . . 306

6.3.4. Abelian deformations ... . . . . . . . . . . . 307

6.4 Calculations for $\mathfrak{s o}_{n}$ : Joseph ideal and highest weight modules . . . . . 307

6.4.1. Solving the constraints . . . . . . . . . . . . . . . 307

6.4.2. Generators of the Joseph ideal . . . . . . . . . . . . . . . . 308

6.4.3. Highest weight module for $\mathfrak{s o}_{n} \ldots \ldots \ldots$. . . . . . . . . 308

6.5 Uniform calculations for the exceptional simple Lie algebras . . . . . . . . 310

6.5.1. Minimal orbits of the exceptional simple Lie algebras . . . . . . . . 310

6.5 .2 . Main result . . . . . . . . . . . . . . . . . . 311

6.5.3. Generators of the Joseph ideal . . . . . . . . . . . . . . . . . 312

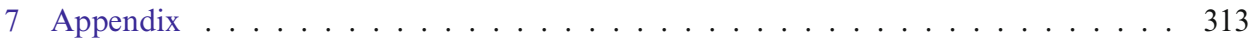

\section{Introduction}

\subsection{GENERAL FRAMEWORK}

Let $\mathfrak{g}$ be a Lie algebra over $\mathbb{C}, \mathfrak{g}^{\prime}$ its $\mathbb{C}$-vector space dual and $G$ its adjoint Lie group. The group acts smoothly on $\mathfrak{g}^{\prime}$ and each orbit comes fitted with a natural symplectic structure (see, e.g. [64]). Moreover, the closure of each coadjoint orbit is an algebraic variety of the type

$$
\left\{\left(x_{1}, \ldots, x_{n}\right) \in \mathbb{C}^{n} ; \forall r \in R, \quad r\left(x_{1}, \ldots, x_{n}\right)=0\right\},
$$

where $R$ is a set of polynomials. We are concerned with the graded coordinate algebra $\mathbb{C}\left[x_{1}, \ldots, x_{N}\right] /\langle R\rangle$. 
As shown by Kirillov, there are interesting relationships between these coadjoint orbits and representation theory; Kostant [59] and Souriau [66] brought in ideas from classical and quantum mechanics and especially from quantization. Meanwhile Gerstenhaber had developed his deformation theory [38], and eventually it was understood [5] that quantization on a symplectic manifold is a deformation of an algebra of functions. One can go further, to regard every such deformation as quantization.

It is curious, we think, that the greater part of recent work on this subject seemed to downplay the special features that coadjoint orbits inherit from the Lie algebra. This paper is a contribution to the study of algebraic invariant starproducts, a type of deformation quantization that makes more intimate contact with Lie structure. Invariant star-products were defined in [5], Definition 4.2.1. We shall borrow from the analogy with quantization of mechanical and field theoretic systems. It turns out that the view of quantization introduced by Weyl, intimately tied to Bohr's correspondence principle, is a most efficient one; it supplements cohomological methods precisely where those become inefficient.

The goal of this paper is not to prove the existence of star-products, invariant or not. In the context of this paper a star-product on a space $S$ is a map from $S \times S$ to $S[[\hbar]]$, or at least an algorithm for evaluating such a map.

The present paper is a new and mathematically more precise version of the original posting by Christian Frønsdal arXiv-math/0510580v1 [math.RT]. It was edited (following Daniel Sternheimer's request to Claude Roger) with Claude Roger and Frédéric Butin.

\subsection{HISTORY OF STAR-PRODUCTS}

A first correspondence between quantum mechanical and classical observables was established by Weyl [70] (classical-to-quantum) and Wigner [71] (the other way). The formula for pulling back the non commutative commutator of operators to classical observables is due to Moyal [62]. The resulting deformation of the Poisson bracket, and of the product of functions on phase space, was subjected to mathematical analysis by Vey [68], following the study of one-differentiable deformations in [26]. The papers [5] generalized this notion and proposed a new axiomatic approach to quantization, interpreted as a formal deformation and formulated in terms of a general type of associative star-product.

One considers the space of formal series, in a parameter $\hbar$, of $\mathcal{C}^{\infty}$ functions on a symplectic manifold $W$, with an associative product of the form

$$
f, g \in \mathcal{C}^{\infty}(W), f * g=f g+\frac{i \hbar}{2}\{f, g\}+\sum_{n=2}^{\infty} \hbar^{n} C_{n}(f, g),
$$

where $f g$ is the ordinary product of functions, $\{\cdot, \cdot\}$ is the Poisson bracket, and the cochains $C_{n}$ are often taken to be bi-differential operators. There were at least four different developments. 
1. The problem of classification of star-products on symplectic spaces, up to a natural but very weak form of equivalence, was investigated by Gutt and others [43]. The existence of star-products on an arbitrary symplectic manifold was established by De Wilde and Lecomte [20] and by Fedosov [21], culminating with the results of Kontsevich who demonstrated the existence of differentiable star-products on an arbitrary Poisson manifold [55,57,67]. The importance of these results is that they are global statements about (smooth) manifolds. Another interesting theorem is due to Cahen et al. [15]: they showed that if $\mathfrak{g}$ is a semi-simple Lie algebra, there is no differential star-product (the coefficients of a differential star product are bidifferential operators) on any neighborhood of the origin in $\mathfrak{g}^{\prime}$ that is tangential to the coadjoint orbits.

2. A generalization of Weyl's original correspondence is required in field theories, "the ordering problem". There have been applications to mechanical problems [2]. Attention is called to the power of this method in the local algebraic context. From this point of view the existence of associative star-products is not surprising, although a concise expression for $f * g$ may be difficult to obtain.

3. The concept of invariant star-products on coadjoint orbits of Lie algebras was proposed in [5]. The existence of invariant star-products on any "regular" coadjoint orbit of a semi-simple Lie algebra was demonstrated in the same paper. This result was obtained by setting up an explicit, equivariant type of Weyl correspondence. The original Weyl correspondence yields, in particular, an invariant star-product for the linear symplectic algebra of the manifold. Invariant star-products are implicit in recent studies of nilpotent orbits, especially those that deal with the Joseph ideal, e.g. [13]. There was an important parallel development in the work of Berezin [8].

4. Invariant star quantization was used as a tool in representation theory, to generalize the method of geometric quantization of Kostant [59] and Souriau [66]. See for example [29,43,69] (and also the book [41] about the Virasoro group for an introduction to geometric quantization). This idea has not yet realized its full potential.

Recently differential and algebraic star-products on regular orbits [24] and on singular orbits $[22,25,60]$ of compact semi-simple Lie groups were studied. These authors study an injective map $[23]\left(\mathbb{C}[X], *_{\text {alg }}\right) \hookrightarrow\left(\mathcal{C}^{\infty}(X), *_{\text {diff }}\right)$, where $X$ is a coadjoint orbit. In the non compact case, they extend their construction of starproducts to singular semi-simple orbits. But the nilpotent singular orbit, that is our main concern here, is not dealt with.

\subsection{TERMINOLOGY}

We recall the definitions of orbits and make precise the terminology that we use throughout the paper. The base field is $\mathbb{C}$ (unless otherwise stated). 
If $\mathfrak{g}$ is a complex semi-simple Lie algebra and $G$ a connected semi-simple Lie group with Lie algebra $\mathfrak{g}$, the coadjoint orbits are the orbits in the dual $\mathfrak{g}^{\prime}$ under the adjoint action of $G$. A coadjoint orbit is said to be semi-simple (resp. nilpotent) if it is the orbit of a semi-simple (resp. nilpotent) element of $\mathfrak{g}^{\prime}$. We will see in Section 5.1 that, unlike the set of semi-simple orbits, the set of nilpotent orbits is finite and contains three canonical orbits, the principal (or regular) orbit, the subregular orbit and the minimal orbit. In this article, we focus our attention on the minimal orbit, denoted $\mathcal{O}_{\min }$. Coadjoint orbits are (smooth) differentiable manifolds. The Zariski closure of the minimal nilpotent orbit is the disjoint union of this orbit and the unique zero dimensional orbit (the origin); it is a singular algebraic variety. In this paper we construct $\mathfrak{g}$-invariant star-products on the Zariski closure $\overline{\mathcal{O}_{\min }}$ of $\mathcal{O}_{\min }$. Since the minimal orbit is determined by its closure, we shall for simplicity often call $\overline{\mathcal{O}_{\min }}$ "the minimal orbit" instead of "the Zariski closure of the minimal orbit". More generally, the closure $\overline{\mathcal{O}}$ of a non-generic orbit $\mathcal{O}$ (a generic orbit is an orbit of maximal dimension) is a singular algebraic variety that is stratified in several orbits of different dimensions.

\subsection{DEFORMATIONS AND HOCHSCHILD COHOMOLOGY}

Since Gerstenhaber [38], it has been clear that the deformations of an algebra of functions on a smooth manifold are classified by its Hochschild cohomology, which is known (for Lie algebras at least since the 70s [26,27,68] and in fact since [47]) to be isomorphic to the space of multivector fields on the manifold. Less well known is the role played by the BGS (Barr-Gerstenhaber-Schack) decomposition of the Hochschild complex. In particular the non existence of (non trivial) abelian deformations in the case of smooth manifolds follows from the vanishing of the Harrison component of Hochschild cohomology. Algebraic varieties offer more room for deformations. The lifting of a Poisson structure to a star-product is governed by components of cohomology that are purely local, associated with the singularities. This strongly suggests that global Poisson structures on varieties more general than smooth manifolds lift to global star-products $[33,56]$. But the results reported here are local.

\subsection{RESULTS OF THE ARTICLE}

This paper is intended as a preliminary study of the algebraic deformations of the coordinate algebras of some algebraic varieties with singularities, a context in which the BGS decomposition $[3,4,28,36,40,46]$ can be expected to have some interesting applications. The coadjoint orbits of simple Lie groups are related by closure to a family of algebraic varieties, and the coordinate algebras of these varieties do in fact have interesting deformations. In that setting our work focuses on invariant star-products to the exclusion of all others.

A familiar reduction paradigm was used to reduce the cohomology to a complex of closed, linear chains. It had been expected that this would lead to an easy 
classification of essential deformations. It turned out, however, to be difficult to obtain enough information about the homology. Before us, experts [13], applying much heavier machinery [7,9] to the same problem, had the same experience and were forced to fall back on heuristic arguments. See below, and in Section 6.1.

Returning to the point of view that sees a star-product as a correspondence between ordinary polynomials and star polynomials, we were able to complete the calculations.

\section{The main results of the paper are the following:}

(a) The Hochschild (co)homology of the graded coordinate algebra of an algebraic variety defined by quadratic relations is isomorphic to that of the restriction to linear, closed chains. Obstructions to extending a first order starproduct (an infinitesimal deformation) to a formal, associative star-product, to all orders in the deformation parameter, can therefore be reduced to a study of the star-products $x * x$ and $x * x * x$ for $x$ of degree 1 .

(b) The minimal, nilpotent, coadjoint orbit of a complex, simple Lie algebra $\mathfrak{g}$ different from $\mathfrak{s l}_{n}$ admits a one-parameter family of invariant star-products/ deformations. In the case of $\mathfrak{s l}_{n}$ we determine a two-parameter family of deformations, including an interesting one-parameter subfamily of abelian deformations. In all the other cases there is a unique, invariant star-product such that

$$
\forall u, v \in \mathfrak{g}^{\prime}, u * v-v * u=\hbar\{u, v\}, \quad \text { and } \quad u * u=u u+k,
$$

for some unique $k \in \mathbb{C}[[\hbar]] ;\{u, v\}$ is the Poisson bracket, see Section 6. The value of $k$ is determined by an examination of the next case,

$$
u * u * u=u u u+\phi(u)
$$

where $\phi$ is a polynomial of degree one. Both $k$ and $\phi$ are uniquely determined by the relations that define the orbit. A uniform calculation covers the five exceptional algebras.

For $\mathfrak{s l}_{n}$ and the other classical simple Lie algebras we calculate the generators of the Joseph ideals and determine the associated highest weight modules. The uniqueness of the Joseph ideal is a corollary; see [35].

\subsection{SOME RECENT RELATED WORKS}

Recently, after arXiv-math/0510580v1 [math.RT] was posted, some reprints appeared that have some relations with it.

In [42], Gukov and Witten study the quantization of a symplectic manifold in the framework of brane theory. They use two-dimensional sigma-models and are mainly interested in the case where the symplectic manifold $M$ admits a complexification $Y$ that is an affine variety, i. e. the set of zeros of a finite subset of 
$\mathbb{C}\left[x_{1}, \ldots, x_{n}\right]$. An important point is the existence of a flat $\operatorname{Spin}_{c}$ structure on $M$. As a consequence of that, they notice in particular that neither their method, nor the one of Brylinski [14], is applicable in the case of the minimal nilpotent orbit of $\mathfrak{s o}(p, q)$ with $p+q$ odd and $p, q \geq 4$. But the results obtained in the present paper for $s l_{n}(\mathbb{C})$ can be applied to that case.

Another interesting study is that of Halbout, Oudom and Tang [44,45]. Their point of view in [45] is different of ours, because they consider a symplectic manifold $M$ and compute the Gerstenhaber bracket of the semi-direct product $\mathcal{C}^{\infty}(M) \rtimes$ $\Gamma$, where $\Gamma$ is a finite group acting on $M$. This allows them to classify the noncommutative Poisson structures on $\mathcal{C}^{\infty}(M) \rtimes \Gamma$. But there is a link with our study. In fact, in the case of minimal singular nilpotent orbits (which we study in this article), if a manifold $M$ is a finite dimensional covering of a singular orbit, the orbit can be represented by a quotient of this covering by a discrete group. As for [44], it is the continuation of [45] in the case of generalized quadratic relations in $T(V) \rtimes \Gamma$, where $T(V)$ is the tensor algebra of a vector space $V$.

\subsection{OUTLINE OF THE PAPER}

Section 2 is an introduction to formal star-products and the BGS decomposition of the Hochschild complex; see [3,4,28,36,40] and [46].

Section 3 is a study of the Hochschild cohomology of algebraic varieties defined by sets of quadratic relations. A main tool used here is a reduction of the Hochschild complex to a subcomplex of closed, linear chains; see Theorem 3.4. The method is effective when the underlying algebra is finitely generated and graded, as it is when the relations are quadratic (homogeneous), with only positive degrees. The BGS decomposition is used throughout.

Section 4 is a brief introduction to invariant star-products on coadjoint orbits of a semi-simple Lie algebra. Section 4.3 gives an example of the appearance of finite representations within the program of star quantization. Included here is the first example (known to us) of harmonic polynomials in the enveloping algebra of a simple Lie algebra. We show an "instance of a deformation" (not a formal deformation) in which the deformed variety (the spectrum of the deformation of the ring of coordinate functions) is a finite union of disconnected varieties.

In Section 5 we study the minimal nilpotent orbits of a semi-simple Lie algebra. We recall some important results about the structure of these orbits. In Section 5.2 we also give the definition of the Joseph ideals. ${ }^{1}$ Then we describe some very special, "unitarizable" representations that are associated with these orbits, and with the Joseph ideals. These representations play a conspicuous role in physics.

Section 6 examines invariant star-products on the most interesting nilpotent coadjoint orbits, those of minimal dimension, with their Joseph ideals. Attempting to calculate the cohomology, we encounter a difficulty that had already been met

\footnotetext{
${ }^{1}$ We thank Birne Binegar for information about Joseph ideals.
} 
by Braverman and Joseph [13], and fail to obtain a sufficiently detailed description of the third homology space $\mathrm{HH}_{3}$ of the coordinate algebra. For the solution of this problem we offer only Conjecture 6.1, but we circumvent the difficulty by an independent, direct calculation. It is done by regarding an invariant star-product as a correspondence principle, in the spirit of Weyl's symmetric ordering. Detailed knowledge of the cohomology of the restricted complex is not needed. Generators of the Joseph ideals are determined.

The Lie algebras $\mathfrak{s l}_{n}$ and $\mathfrak{s o}_{n}$ are treated separately and all the calculations are included, with some proofs relegated to an Appendix. The case of $\mathfrak{s p}_{2 n}$ is too well known to warrant much attention. The five exceptional simple Lie algebras are handled uniformly together, all the calculations are in the main text, they are both short and easy, because of the small number of invariants of these Lie algebras.

Within a family of generally non commutative, invariant star-products there may be a subfamily of non trivial abelian ones. According to Braverman and Joseph, this would contradict the fact that the minimal orbits - excepting the case of $\mathfrak{s l}_{n}$ are rigid. Granted that a deformation of the ring of coordinate functions implies a deformation of its spectrum. But is it known that the deformed spectrum of an abelian deformation is always an algebraic variety? ${ }^{2}$ Is every equivariant deformation of a coadjoint orbit a coadjoint orbit? In the event, we confirm that abelian deformations of the coordinate algebra exist only in the case of $\mathfrak{s l}_{n}$, and that in that case the spectrum can be identified with a neighboring orbit of the same dimension.

\section{Star-products and Hochschild (Co)Homology}

\subsection{HOCHSCHILD (CO)HOMOLOGY AND DEFORMATIONS}

- Given an associative and commutative $\mathbb{C}$-algebra, denoted by $A$, the homological reduced Hochschild chain complex of $A$ is

${ }^{2}$ We are grateful to Dmitry Kaledin for the following instructive remarks on that question. Given an algebraic variety $X$ and a one-parameter commutative deformation $\mathcal{O}_{\hbar}$ of the structure sheaf $\mathcal{O}_{X}$, one can ask whether it is true that there exists an algebraic variety $Y$ containing $X$ such that sections of $\mathcal{O}_{\hbar}$ are formal germs of functions on $Y$ near $X$. The answer to this depends on what one understands by an "algebraic variety". If one just means "scheme", and $X$ is affine, then the answer is yes for trivial reasons (take $Y=\operatorname{Spec}_{0}\left(X, \mathcal{O}_{\hbar}\right)$ ). However, usually one wants varieties to be "nice"-for example, schemes of finite type (algebraically, finitely generated algebras). If this is what one wants from $Y$, then the problem is non-trivial; in some cases the answer is still yes, but this requires heavy machinery such as Grothendieck's algebraization theorem for formal schemes. Furthermore, in reference to results of Braverman-Joseph on rigidity of nilpotent orbits, it is reasonable to understand by "variety" something to which rigidity applies. And then, there is another context where there are no problems: one can replace a (closed) orbit $X$ by its completion at 0 and treat it as a Noetherian local formal scheme (algebraically, this corresponds to working with $m$-adically complete local algebras $(A, m)$ such that the associated graded quotient $\operatorname{gr} A=\bigoplus_{i}\left(\mathrm{~m} / \mathrm{m}^{2}\right)^{i}$ is finitely generated). Then $Y$ also trivially exists as a Noetherian formal local scheme, and the rigidity still applies. 


$$
\cdots \stackrel{d_{5}}{\longrightarrow} \mathrm{C}_{4}(A) \stackrel{d_{4}}{\longrightarrow} \mathrm{C}_{3}(A) \stackrel{d_{3}}{\longrightarrow} \mathrm{C}_{2}(A) \stackrel{d_{2}}{\longrightarrow} \mathrm{C}_{1}(A),
$$

where the $p$-chains are defined by $\mathrm{C}_{p}(A)=A^{\otimes p}$. The differential $d_{\bullet}=\bigoplus_{p=1}^{\infty} d_{p}$ is given by $d_{1}(a)=0$ and for $p>1$

$$
\begin{aligned}
d_{p}\left(a_{1} \otimes a_{2} \otimes \cdots \otimes a_{p}\right)= & a_{1} a_{2} \otimes a_{3} \otimes \cdots \otimes a_{p}+ \\
& +\sum_{i=2}^{p-1}(-1)^{i+1} a_{1} \otimes a_{2} \otimes \cdots \otimes a_{i} a_{i+1} \otimes \cdots \otimes a_{p} .
\end{aligned}
$$

Then we define the Hochschild homology of $A$ by

$$
\mathrm{Z}_{p}(A)=\operatorname{Ker} d_{p}, \mathrm{~B}_{p}(A)=\operatorname{Im} d_{p+1} \text { and } H H_{p}(A)=\mathrm{Z}_{p}(A) / \mathrm{B}_{p}(A) .
$$

- Similarly, the cohomological Hochschild complex of $A$ is the following

$$
\mathrm{C}^{0}(A) \stackrel{d^{(0)}}{\longrightarrow} \mathrm{C}^{1}(A) \stackrel{d^{(1)}}{\longrightarrow} \mathrm{C}^{2}(A) \stackrel{d^{(2)}}{\longrightarrow} \mathrm{C}^{3}(A) \stackrel{d^{(3)}}{\longrightarrow} \mathrm{C}^{4}(A) \stackrel{d^{(4)}}{\longrightarrow} \cdots,
$$

where the space $\mathrm{C}^{p}(A)$ of $p$-cochains is, for $p \in N^{*}$, the space of $\mathbb{C}$-linear maps from $A^{\otimes p}$ to $A$, and $C^{0}=A$. The differential $d^{\bullet}=\bigoplus_{p=0}^{\infty} d^{(p)}$ is given by

$$
\begin{aligned}
\forall C \in \mathrm{C}^{p}(A), d^{(p)} C\left(a_{0}, \ldots, a_{p}\right)= & a_{0} C\left(a_{1}, \ldots, a_{p}\right)-C\left(d_{p+1}\left(a_{0} \otimes \cdots \otimes a_{p}\right)\right)+ \\
& +(-1)^{p-1} C\left(a_{0}, \ldots, a_{p-1}\right) a_{p} .
\end{aligned}
$$

We may write it in terms of Gerstenhaber $\operatorname{bracket}^{3}[\cdot, \cdot]_{G}$ and the product $\mu$ of $A$, as follows:

$$
d^{(p)} C=(-1)^{p+1}[\mu, C]_{G} .
$$

\footnotetext{
${ }^{3}$ Recall that for $F \in \mathrm{C}^{p}(A)$ and $H \in \mathrm{C}^{q}(A)$, the Gerstenhaber product is the element $F \bullet H \in$ $\mathrm{C}^{p+q-1}(A)$ defined by

$$
F \bullet H\left(a_{1}, \ldots, a_{p+q-1}\right)=\sum_{i=0}^{p-1}(-1)^{i(q+1)} F\left(a_{1}, \ldots, a_{i}, H\left(a_{i+1}, \ldots, a_{i+q}\right), a_{i+q+1}, \ldots, a_{p+q-1}\right) .
$$
}

It is not associative, but the associator $\operatorname{Ass}(F, H, K):=(F \bullet H) \bullet K-F \bullet(H \bullet K)$ verifies the relation

$$
\operatorname{Ass}(F, H, K)=(-1)^{(q-1)(r-1)} \operatorname{Ass}(F, K, H) .
$$

Therefore, the Gerstenhaber bracket, defined by $[F, H]_{G}:=F \bullet H-(-1)^{(p-1)(q-1)} H \bullet F$, is antisymmetric,

$$
[F, H]_{G}=-(-1)^{(p-1)(q-1)}[H, F]_{G},
$$

and satisfies the graded Jacobi identity

$$
\left[F,[H, K]_{G}\right]_{G}+(-1)^{(p-1)(q+r-2)}\left[H,[K, F]_{G}\right]_{G}+(-1)^{(r-1)(p+q-2)}\left[K,[F, H]_{G}\right]_{G}=0 .
$$

See for example [37], and [16, p. 38]. 
Then we define the Hochschild cohomology of $A$ by $H H^{0}(A)=\operatorname{Ker} d^{(0)}=A$ and

$$
\begin{aligned}
& \forall p \in \mathbb{N}^{*}, \quad Z^{p}(A)=\operatorname{Ker} d^{(p)}, \quad \mathrm{B}^{p}(A)=\operatorname{Im} d^{(p-1)}, \quad \text { and } \\
& H H^{p}(A)=Z^{p}(A) / \mathrm{B}^{p}(A) .
\end{aligned}
$$

This paper makes use of a pairing between homology and cohomology that requires that we deal with the restriction of $A$ to a non-unital subalgebra $A_{+}$. (Section 3.1.)

We denote by $\mathbb{C}[[\hbar]]$ (resp. $A[[\hbar]]$ ) the algebra of formal power series in the parameter $\hbar$, with coefficients in $\mathbb{C}$ (resp. $A$ ). A star-product of the algebra $A$ is defined as a map $*$ from $A[[\hbar]] \times A[[\hbar]]$ to $A[[\hbar]]$ that is $\mathbb{C}[[\hbar]]$-bilinear and such that, $\forall k \in \mathbb{C}, f \in A$,

$$
k * f=f * k=f,
$$

and $\forall f, g, h \in A$,

$$
f * g \equiv f g \quad \bmod \hbar A[[\hbar]], \quad f *(g * h)=(f * g) * h .
$$

This means that there exists a sequence of bilinear maps ${ }^{4} C_{j}$ from $A \times A$ to $A$ of which the first term $C_{0}$ is the product of $A$ and such that

$$
\begin{aligned}
& \forall a, b \in A, \quad a * b=\sum_{j=0}^{\infty} C_{j}(a, b) \hbar^{j}, \\
& \forall n \in \mathbb{N}, \quad \sum_{i+j=n} C_{i}\left(a, C_{j}(b, c)\right)= \\
& =\sum_{i+j=n} C_{i}\left(C_{j}(a, b), c\right), \text { that is } \sum_{i+j=n}\left[C_{i}, C_{j}\right]_{G}=0 .
\end{aligned}
$$

We call this a star-product of order $p$ if the Equation (2) is satisfied (only) for $n \leq p$.

A first order star-product is a product

$$
f * g=f g+\hbar C_{1}(f, g)
$$

associative to first order in $\hbar$, which makes $C_{1}$ a closed Hochschild cochain, namely

$$
d^{(2)} C_{1}(f, g, h):=f C_{1}(g, h)-C_{1}(f g, h)+C_{1}(f, g h)-C_{1}(f, g) h=0 .
$$

If $C_{1}$ is exact, that is, if there is a 1 -cochain such that

$$
C_{1}(f, g)=d^{(1)} E(f, g),
$$

\footnotetext{
${ }^{4}$ Often, in the definition of a star-product (see for example [16]), the bilinear maps $C_{j}$ 's are bidifferential operators. Here, we do not make this assumption.
} 
then, to first order in $\hbar$, Equation 3 can be written

$$
(f-\hbar E(f)) *(g-\hbar E(g))=f g-\hbar E(f g) .
$$

Essential first order deformations are classified by $H H^{2}(A)$ (see [37]).

If $m=\sum_{j=0}^{p} C_{j} \hbar^{j}$ is a star-product of order $p$, then we may extend $m$ to a starproduct of order $p+1$ if and only if there exists $C_{p+1}$ such that $\forall a, b, c \in A$,

$$
\begin{aligned}
& \sum_{i=1}^{p}\left(C_{i}\left(C_{p+1-i}(a, b), c\right)-C_{i}\left(a, C_{p+1-i}(b, c)\right)\right)=d^{(2)} C_{p+1}(a, b, c), \\
& \sum_{i=1}^{p}\left[C_{i}, C_{p+1-i}\right]_{G}=2 d^{(2)} C_{p+1} .
\end{aligned}
$$

The left hand side is closed; this minor miracle is responsible for the fact that commutative algebras are not isolated in the family of associative algebras. (For this easy but important fact, see for example $[5,16,38])$. So $H H^{3}(A)$ contains the obstructions to extend a deformation of order $p$ to a deformation of order $p+1$.

\subsection{THE BGS DECOMPOSITION OF HOCHSCHILD (CO)HOMOLOGY}

In this section, we explain how the Hochschild cohomological complex splits into a finite or infinite sum of direct summands. This decomposition is based on the action of the symmetric group $\mathfrak{S}_{n}$ on $n$-cochains, and on the existence of idempotents. In the case where the algebra is generated by $N$ generators, there are only $N$ nonzero summands. The complete decomposition of the Hochschild cohomology of a commutative algebra was found by Gerstenhaber and Schack [40], after the pioneering work of Harrison [46] and Barr [3,4,39] (See also [61]).

Consider the rational group algebra $\mathbb{Q}\left(\mathfrak{S}_{n}\right)$, and let $L\left(\mathrm{C}^{(n)}(A)\right)$ be the algebra of linear maps from $C^{(n)}(A)$ to $C^{(n)}(A)$. There is a natural injective morphism

$$
\mathbb{Q}\left(\mathfrak{S}_{n}\right) \hookrightarrow L\left(\mathrm{C}^{(n)}(A)\right)
$$

that allows us to identify the elements of $\mathbb{Q}\left(\mathfrak{S}_{n}\right)$ with linear maps from $\mathrm{C}^{(n)}(A)$ to $\mathrm{C}^{(n)}(A)$. The following result is the key point for the decomposition of the Hochschild cohomology.

THEOREM 2.1. (Barr-Gerstenhaber-Schack) For each $n \geq 2$, there exist $n$ elements $e_{n}(1), \ldots, e_{n}(n)$ of $\mathbb{Q}\left(\mathfrak{S}_{n}\right)$ that verify the following properties:

(1) $e_{n}(k)^{2}=e_{n}(k)$,

(2) $e_{n}(k) e_{n}(l)=0$ for $k \neq l$ (the $e_{n}(k)$ 's are mutually orthogonal),

(3) $\sum_{k=1}^{n} e_{n}(k)=1$,

(4) $d^{(n)} \circ e_{n}(k)=e_{n+1}(k) \circ d^{(n)}$. 
The elements $e_{n}(k)$ defined above are called BGS idempotents or Eulerian idempotents. It is interesting to note that these elements are some Lagrange interpolation polynomials. See [4] and [40] for the proof of Theorem 2.1 and for more details about the BGS idempotents.

There are thus decompositions

$$
\mathrm{C}^{n}=\bigoplus_{k=1}^{n} \mathrm{C}^{n, k}, \quad \mathrm{Z}^{n}=\bigoplus_{k=1}^{n} \mathrm{Z}^{n, k}, \quad \mathrm{~B}^{n}=\bigoplus_{k=1}^{n} \mathrm{~B}^{n, k}
$$

of the spaces of $n$-cochains, $n$-cocycles and $n$-coboundaries, and we have the BGS decomposition

$$
H H^{n}=\bigoplus_{k=1}^{n} H H^{n, k}
$$

with $\operatorname{Harr}^{n}:=H H^{n, 1}$.

A generating function for the idempotents was found by Garsia [36]:

$$
\sum_{k=1}^{n} z^{k} e_{n}(k)=\frac{1}{n !} \sum_{\sigma \in \mathfrak{S}_{n}}\left(z-\delta_{\sigma}\right)\left(z-\delta_{\sigma}+1\right) \cdots\left(z-\delta_{\sigma}+n-1\right)(-1)^{\sigma} \sigma,
$$

where $(-1)^{\sigma}$ is the sign of $\sigma$ and $\delta_{\sigma}$ is the number of descents of $\sigma, \delta_{\sigma}=$ $|\{(i, i+1) ; \sigma(i)>\sigma(i+1)\}|$. For example, the permutation (1342) has two descents, from 1 to 2 and from 3 to 4 .

The simplest idempotents are

$$
\begin{aligned}
& e_{2}(1) 12=\frac{1}{2}(12+21), \\
& e_{2}(2) 12=\frac{1}{2}(12-21), \\
& e_{3}(1) 123=\frac{1}{6}(2(123-321)+132-231+213-312), \\
& e_{3}(2) 123=\frac{1}{2}(123+321), \\
& e_{n}(n)=\frac{1}{n !} \sum_{\sigma \in \mathfrak{S}_{n}}(-1)^{\sigma} \sigma .
\end{aligned}
$$

The spaces of Hochschild $n$-chains, $n$-cycles and $n$-boundaries decompose in the same way, with

$$
d_{n} \circ e_{n}(k)=e_{n-1}(k) \circ d_{n},
$$

and

$$
\mathrm{C}_{n}=\bigoplus_{k=1}^{n} \mathrm{C}_{n, k}, \quad \mathrm{Z}_{n}=\bigoplus_{k=1}^{n} \mathrm{Z}_{n, k}, \quad \mathrm{~B}_{n}=\bigoplus_{k=1}^{n} \mathrm{~B}_{n, k},
$$


so that

$$
H H_{n}=\bigoplus_{k=1}^{n} H H_{n, k},
$$

with $\operatorname{Harr}_{n}:=H H_{n, 1}$.

\subsection{APPLICATION OF THE BGS DECOMPOSITION TO STAR-PRODUCTS}

According to the preceding section, every 2-cochain $C$ has a decomposition

$$
C=e_{2}(1)(C)+e_{2}(2)(C)=C^{+}+C^{-} \quad \text { with } \quad C^{+} \in \mathrm{C}^{2,1} \quad \text { and } \quad C^{-} \in \mathrm{C}^{2,2} ;
$$

that is, $C^{+}$(resp. $C^{-}$) is a symmetric (resp. skew-symmetric) bilinear map.

Associativity of the star-product Equation (1) to first order is the requirement that both 2-cochains be closed, $d^{(2)} C^{+}=d^{(2)} C^{-}=0$. A first order deformation of the algebra is inessential if both forms are exact. In the case of a smooth manifold, $\mathrm{Harr}^{2}$ is empty and choosing $\mathrm{C}_{1}^{+}=0$ entails no essential loss.

Returning now to Equation (4), we have mappings

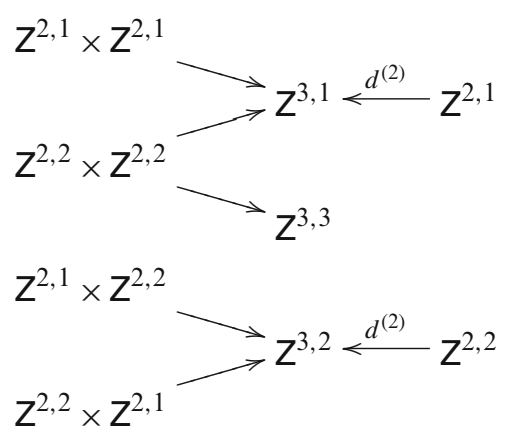

where the first column of arrows represents the construction on the left hand side of Equation (4) (with the Gerstenhaber bracket) and the second column of arrows is the mapping by the differential $d^{(2)}$.

Because $\mathrm{B}^{3,3}$ is empty, the obstruction in $\mathrm{Z}^{3,3}=H H^{3,3}$ demands that the skewsymmetric part of the left hand side of Equation (4) vanish. That is the Jacobi identity, satisfied if $C_{1}^{-}$is a Poisson bracket. The first line shows that the obstruction to abelian deformations is the Harrison component $\operatorname{Harr}^{3}=H H^{3,1} \subset H H^{3}$.

In the case of smooth manifolds $H H^{n}=H H^{n, n}=0$, and abelian deformations are inessential, according to the HKR Theorem. The familiar deformations with $C_{1}=C_{1}^{-}$encounter no additional obstructions to second order because, if $C_{1}^{+}=0$, the brackets of the first column belong to $Z^{3,1}$ and $Z^{3,3}$. But $H H^{3,3}=0$, so the only obstruction is $\mathrm{Harr}^{3}$.

We now turn to a preliminary investigation of algebraic varieties with singularities. We shall find varieties for which $Z^{3,1}, Z^{3,2}$ and $Z^{3,1}$ are all non empty. 


\section{Hochschild (Co)Homology of Some Varieties with Singularities}

\subsection{CASE OF QUADRATIC RELATIONS: THE CONIC VARIETIES}

We call conic variety an algebraic variety of the type $\mathbb{C}^{N} / R$, where $R=\left\{g_{\alpha}\right\}_{\alpha=1,2, \ldots}$ is a set of homogeneous, quadratic polynomials,

$$
g_{\alpha}=\sum_{i, j=1}^{N} g_{\alpha}^{i j} x_{i} x_{j} .
$$

Let $A$ be the graded coordinate algebra, $A=\mathbb{C}\left[x_{1}, \ldots, x_{N}\right] /\langle R\rangle$, and $A_{+}$the subalgebra of elements of positive degree (so we shall use the reduced Hochschild complex, called here for simplicity the Hochschild complex). The restriction to positive degrees is essential: in fact, cochains on $A_{+}$extend naturally to $A$, but the homology of $A_{+}$is richer than that of $A$ (the homology of $A$ is zero). In this section, we make use of some results of [30].

DEFINITION 3.1. A Hochschild chain $a=a_{1} \otimes a_{2} \otimes \cdots \otimes a_{p}$ will be said to be linear if $a_{k}$ is of degree 1 for every $k \in\{1, \ldots, p\}$.

The restricted homological Hochschild complex is the restriction of the homological Hochschild complex of $A$ to closed, linear chains. We denote by $\widetilde{\boldsymbol{C}}_{p}(A)$ the space of $p$-chains of the restricted complex.

The differential of this complex is zero, so that the homology space of degree $p$ is $\widetilde{C}_{p}(A)$ itself.

Remark 3.2. In the case of a smooth manifold, the chains of the restricted complex are the skew-symmetric ones; the next theorem reduces in that case to the well-known result of Hochschild, Kostant and Rosenberg [47]. For other generalizations see [32,33], and Section 3.3.

DEFINITION 3.3. Given two complexes, a quasi-isomorphism between them is a morphism of complexes that induces an isomorphism in cohomology.

THEOREM 3.4. The Hochschild complex of A is quasi-isomorphic to the (co)homological restricted complex.

Proof. (see also [30].)

The restriction of a closed/exact form is closed/exact. Conversely, every closed/exact restricted cochain is the restriction of a closed/exact Hochschild cochain. It is enough to consider homogeneous chains; that is, $a=a_{1} \otimes \cdots \otimes a_{n}$ such that each factor $a_{k}, k=1, \ldots, n$ is of well defined degree. (The only grading that we use is the total polynomial degree.) To show that every restricted (=closed, linear) $n$-cochain extends to a closed, Hochschild $n$-cochain we consider the formula 


$$
d^{(n)} C\left(a_{1}, \ldots, a_{n+1}\right)=a_{1} C\left(a^{1}\right)-C\left(d_{n} a\right)+(-1)^{n+1} C\left(a^{n+1}\right) a_{n+1},
$$

where $a^{1}=a_{2} \otimes \cdots \otimes a_{n+1}$ and $a^{n+1}=a_{1} \otimes \cdots \otimes a_{n}$. Evidently the degree of $a$ is higher than the degrees of $a^{1}$ and $a^{n+1}$. This formula can therefore be used to try to extend closedness, recursively to higher degrees. The obstruction is $d_{n} a=0$, but it is easy to verify that the remaining terms in Equation (6) vanish when $a$ is exact and $C$ is closed on lower degrees. The obstruction comes from homology; if the restricted $n$-cochain $C$ has the property that $d^{(n)} C(h)=0$ for a representative $h$ of every homology class of $(n+1)$-chains, then it extends to a closed, Hochschild $n$-cochain.

In the absence of singularities the restricted cochains are alternating and the cochains are defined on alternating chains only. These $n$-cochains are closed and not homologous to zero because the differential does not connect the projections $e_{n}(n)$ and $e_{(n+1)}(n+1)$. That is, the differential is zero. In the presence of singularities there are additional, homologically non trivial chains, and the closedness of a cochain, even if alternating, has to be tested on them. There is a net loss of alternating cohomology and non zero cohomology in the other BGS sectors.

\subsection{CASE OF ONE QUADRATIC RELATION: THE SIMPLE CONE}

In this section, we study a particular case of conic variety. We retain all the definitions but we suppose that $R=\{g\}$ is just one quadratic polynomial. In this case the following holds.

PROPOSITION 3.5. Every closed chain is homologous to a linear chain and no linear chain is exact. The space $Z_{2 k+l}$ of closed, linear $(2 k+l)$-chains is spanned by the following $(2 k+l)$-chains, with $Z_{2 k+l, k+l} \in Z_{2 k+1, k+l}$ :

$$
\begin{gathered}
\left(Z_{2 k+l, k+l}\right)_{m_{1} \ldots m_{l}}=g^{i_{1} j_{1}} \ldots g^{i_{k} j_{k}} \sum_{\sigma}(-1)^{\sigma} \sigma\left(x_{i_{1}} \otimes \cdots \otimes x_{j_{k}} \otimes x_{m_{1}} \otimes \cdots \otimes x_{m_{l}}\right), \\
\forall m_{1}, \ldots, m_{l}=1, \ldots, N,
\end{gathered}
$$

where the sum is over all permutations of the set $\left\{i_{1}, \ldots, i_{k}, j_{1}, \ldots, j_{k}, m_{1}, \ldots, m_{l}\right\}$ that preserve the internal order of each pair $\left(i_{1}, j_{1}\right),\left(i_{2}, j_{2}\right), \ldots,\left(i_{k}, j_{k}\right)$.

EXAMPLE 3.6.

$$
\begin{aligned}
\left(Z_{1,1}\right)_{i} & =x_{i}, \quad i=1 \ldots N, \\
\left(Z_{2,2}\right)_{i j} & =x_{i} \wedge x_{j}, \\
Z_{2,1} & =g^{i j} x_{i} \otimes x_{j}, \\
\left(Z_{3,3}\right)_{i j k} & =x_{i} \wedge x_{j} \wedge x_{k}, \\
\left(Z_{3,2}\right)_{k} & =g^{i j}\left(x_{i} \otimes x_{j} \otimes x_{k}-x_{i} \otimes x_{k} \otimes x_{j}+x_{k} \otimes x_{i} \otimes x_{j}\right),
\end{aligned}
$$




$$
\begin{aligned}
\left(Z_{4,4}\right)_{i j k l}= & x_{i} \wedge x_{j} \wedge x_{k} \wedge x_{l}, \\
\left(Z_{4,3}\right)_{k l}= & g^{i j}\left(x_{i} \otimes x_{j} \otimes x_{k} \otimes x_{l}+x_{k} \otimes x_{i} \otimes x_{j} \otimes x_{l}+x_{i} \otimes x_{l} \otimes x_{j} \otimes x_{k}+\right. \\
& \left.+x_{k} \otimes x_{l} \otimes x_{i} \otimes x_{j}+x_{i} \otimes x_{k} \otimes x_{l} \otimes x_{j}+x_{l} \otimes x_{i} \otimes x_{k} \otimes x_{j}-(k \leftrightarrow l)\right), \\
\left(Z_{4,2}\right)= & g^{i j} g^{k l}\left(x_{i} \otimes x_{k} \otimes x_{l} \otimes x_{j}-x_{i} \otimes x_{k} \otimes x_{j} \otimes x_{l}+x_{i} \otimes x_{j} \otimes x_{k} \otimes x_{l}\right) .
\end{aligned}
$$

From this point onwards, the notation $\mathrm{Z}_{k, l}, \mathrm{~B}_{k, l}, \mathrm{Z}^{k, l}, \mathrm{~B}^{k, l}$ stands for spaces defined with reference to the restricted complex. The dimension of $\mathbf{Z}_{2 k+l, k+l} \subset$ $H H_{2 k+l, k+l}$ is $\left(\begin{array}{l}l \\ N\end{array}\right)$ or 0 . We note that $Z_{3,1}$ is empty. To lowest order, a star-product is determined by the 2-cochain $C_{1}$, and up to equivalence by the restricted 2-cochains; that is, by their values $C_{1}^{-}\left(x_{i} \wedge x_{j}\right)$ and $g^{i j} C_{1}^{+}\left(x_{i}, x_{j}\right)$ on the homological basis. The differential $d^{(2)} C_{1}^{+}$is in $\mathrm{B}^{3,1}$, and since $\mathrm{Z}^{3,1}$ is empty, every restricted, symmetric 2-cochain is closed. The differential $d^{(2)} C_{1}^{-}$is in $\mathrm{B}^{3,2} ; C_{1}^{-}$is closed if and only if $d^{(2)} C_{1}^{-}$vanishes on $Z_{3,2}$,

$$
d^{(2)} C_{1}^{-}\left(Z_{3,2}\right)=4 g^{i j} x_{i} C_{1}^{-}\left(x_{j}, x_{k}\right)=0 .
$$

This will be interpreted as the statement that the "hamiltonian" vector field $C_{1}^{-}\left(x_{k}, \cdot\right)$ is tangential to the constraint surface.

The addition of an exact form $d^{(2)} E$ to $C_{1}$ (we like to think of it as a 'gauge transformation') does not affect $C_{1}^{-}$but it adds $2 g^{i j} x_{i} E\left(x_{j}\right)$ to $C_{1}^{+}\left(Z_{2,1}\right)=$ $g^{i j} C_{1}^{+}\left(x_{i}, x_{j}\right)$.

EXAMPLE 3.7. Suppose that the 2-form $g$ is non degenerate; then $C_{1}^{+}$is fixed up to equivalence by its degree zero term (in the case of regular functions on the closure, by its value $c=\left.g^{i j} C_{1}^{+}\left(x_{i}, x_{j}\right)\right|_{x=0}$ at $\left.x_{1}=x_{2}=\cdots=0\right)$. That is, $H H^{2,2}$ is the space of tangential vector fields on the cone and $H H^{2,1}=\mathbb{C}$. (See the end of Section 3.2 for equivariant cohomology.)

We examine the obstructions to extending the star-product to all orders in $\hbar$, referring to Equations (4) and (5).

The emptiness of $\mathrm{B}^{3,3}$ is an obstruction that must be circumvented by imposing the Jacobi identity on $C_{1}^{-}$. Because $C_{1}^{-}$is closed this entails that it extends to a unique Poisson bracket on $A$. Recall that, if $(a, b) \mapsto\{a, b\}$ is a Poisson bracket on $A$ then for every $f \in A$, the mapping $f^{\sharp}: A \rightarrow A$ defined by $a \mapsto\{f, a\}$ is a derivation. The projection of Equation (4) on $\mathrm{Z}_{2,3}$ is now solved by taking $C_{n}^{-}\left(x_{i}, x_{j}\right)=$ $0, \forall i, j=1 \ldots N$ and $\forall n \geq 2$. This choice is implicit in the context of differentiable deformations, and it is one of the axioms of invariant star-products. We do not investigate alternatives.

- Summary: The projection of Equation (5) on $Z_{3,3}$ leads to

(1) $C_{1}^{-}\left(x_{i}, x_{j}\right)=\left\{x_{i}, x_{j}\right\}$ extends to a Poisson bracket on $A$,

(2) $\left\{x_{i}, g(x, x)\right\}=0$ is the condition that $C_{1}$ be closed, 
(3) $C_{n}^{-}\left(x_{i}, x_{j}\right)=0 \forall i, j=1 \ldots N$ and $\forall n \geq 2$.

The projection of Equation (4) on $Z_{3,2}$ takes the form, for $k=1, \ldots, N$,

$$
\begin{aligned}
& \sum_{m+n=p+1}\left[-4 g^{i j} C_{m}^{+}\left(C_{n}^{-}\left(x_{i}, x_{k}\right), x_{j}\right)+2 g^{i j} C_{m}^{-}\left(C_{n}^{+}\left(x_{i}, x_{j}\right), x_{k}\right)\right]= \\
& \quad=d^{(2)} C_{p+1}^{-}\left(Z_{3,2}\right)_{k}=4 g^{i j} x_{i} C_{p+1}^{-}\left(x_{j}, x_{k}\right) .
\end{aligned}
$$

If $g$ is non degenerate, then we can restrict the value of $C_{1}^{+}\left(x_{i}, x_{j}\right)$ to $\mathbb{C}$; see Example 3.7. The obstruction is then the term of degree zero on the left hand side. In view of Summary (3) above, Equation (7) simplifies to

$$
2 g^{i j} C_{p}^{+}\left(\left\{x_{i}, x_{k}\right\}, x_{j}\right)-g^{i j}\left\{C_{p}^{+}\left(x_{i}, x_{j}\right), x_{k}\right\}=0 .
$$

EXAMPLE 3.8. If $\left\{x_{i}, x_{k}\right\}=\varepsilon_{i k}^{m} x_{m}$, the coefficients $\varepsilon_{i k}^{m}$ being the structure constants of a simple Lie algebra $\mathfrak{g}$, then Equation (8) is satisfied when $C_{p}^{+}$is the Killing form of $\mathfrak{g}$.

EXAMPLE 3.9. In the case of $\mathfrak{s l}_{n}$, there is a well-known equivariant 3-tensor $f: A \otimes A \rightarrow A$ and $C_{p}^{+}=f$ also solves Equation (8). Explicitly, we have $f(a \otimes b)=$ $a b+b a-\frac{2}{n} \operatorname{tr}(a b) I_{n}$.

EXAMPLE 3.10. Choose coordinates such that $g(x, x)=x_{N}^{2}-\rho(x)$, where $\rho(x)$ is a polynomial in $x_{1}, \ldots, x_{N}$, at most linear in $x_{N}$. A regular function on the cone, being the restriction of a polynomial in $x_{1}, \ldots, x_{N}$, has a unique decomposition of the form $f=f_{1}+x_{N} f_{2}$, where $f_{1}, f_{2}$ are polynomials in $x_{1}, \ldots, x_{N-1}$. Define ([31])

$$
f * g=f g+\hbar f_{2} g_{2}=\left.f g\right|_{x_{N}^{2}=\rho(x)+\hbar} .
$$

Then the bilinear map $*$ is a star-product; it can be interpreted as the ordinary product of functions on the hyperboloid $g(x, x)=\hbar$. This deformation, in which $C_{1}$ is symmetric, can be followed by another deformation in which $C_{1}$ is skew-symmetric, leading to a Poisson bracket such that the vector fields $\left\{x_{i}, \cdot\right\}$; are tangent to the hyperboloid $g(x, x)=\hbar$; no Harrison cohomology intervenes in either stage. When both deformations are combined we note that, with $C_{1}^{+}$as we have defined it in Equation (9), $C_{1}^{+}(f, g)=f_{2} g_{2}$, the only contribution to the left hand side of Equation (8) at $x=0$ comes from the linear term in $\left\{x_{i}, x_{k}\right\}$. Closure of $C_{m}^{-}$implies that the vector field $\left\{\cdot, x_{k}\right\}$ is tangent to $g(x, x)=\hbar$.

- Equivariant cohomology: in the context of Lie algebras and invariant star-products all maps will be equivariant. This affects the question of exactness, as in Example 3.7, and will be taken into account as the occasion arises. Theorem 3.4 is not affected. 


\subsection{CASE OF ONE POLYNOMIAL RELATION}

Let $A=\mathbb{C}\left[x_{1}, \ldots, x_{N}\right] /\langle g\rangle$, where $g$ is a polynomial without constant or linear terms.

Grading: we can no longer restrict our attention to homogeneous chains. By an appropriate linear transformation of variables we can bring the polynomial $g$ to the form $x_{1}^{t}+h\left(x_{1}, \ldots, x_{N}\right)$, where $h$ is a polynomial of degree less than $t$ in $x_{1}$.

A normalized polynomial is one that is of degree less than $t$ in $x_{1}$; to each regular function on $\mathbb{C} / g$ there is just one normalized polynomial and the degree of a regular function is defined to be the degree of this normalized polynomial. The normalized polynomial is obtained by a Euclidean division in $\left(\mathbb{C}\left[x_{2}, \ldots, x_{N}\right]\right)\left[x_{1}\right]$. Let $g$ be a normalized polynomial in $x_{1}, \ldots, x_{n}$, without a constant term and without linear terms. Choose a presentation

$$
g=\sum_{a, b=1}^{K} g^{a b} y_{a} y_{b}, \text { with } g^{a b}=g^{b a} \in \mathbb{C}, a, b=1 \ldots K,
$$

where for each $a=1 \ldots K$, the polynomial factor $y_{a}$ has no constant term, and let $A$ be the filtered algebra $A=\mathbf{C}\left[x_{1}, \ldots, x_{N}\right] /\langle g\rangle$ with $A_{+}$the sum of the positive degrees of $A$. With each element of $A_{+}$is associated a unique, normalized polynomial.

Let $Z_{2 k+l}$ denote the space of closed $p$-chains, spanned by the following $p$-chains, for $p=2 k+l=1,2 \ldots$, and $m_{1}, \ldots, m_{l}=1, \ldots, N$,

$$
\left(Z_{2 k+l, k+l}\right)_{m_{1} \ldots m_{l}}=g^{a_{1} b_{1}} \ldots g^{a_{k} b_{k}} \sum_{\sigma}(-)^{\sigma} \sigma\left(y_{a_{1}} \otimes \cdots \otimes y_{b_{k}} \otimes x_{m_{1}} \otimes \cdots \otimes x_{m_{l}}\right),
$$

where the sum is over all permutations that preserve the internal order of each pair $\left(a_{1}, b_{1}\right), \ldots,\left(a_{k}, b_{k}\right)$.

THEOREM 3.11. The restriction of the Hochschild complex to such chains is a quasi-homomorphism; that is, the cohomologies are isomorphic.

The proof of this theorem is as the proof of Theorem 3.4, but it needs the following lemma. Let $A_{+}$be the filtered algebra without unit as above. The degree of an $A_{+}$-chain $a_{1} \otimes \cdots \otimes a_{p}$ is the sum of the degrees of its factors.

LEMMA 3.12. If an $A_{+} p$-chain $a$ is exact, then there is $a(p+1)$-chain $b$, with the same degree as a, such that $a=d_{p+1} b$. See [33]. 


\section{Introduction to g-Invariant Star-Products on Coadjoint Orbits}

\subsection{BACKGROUND}

The origin of the problem is as follows (see [1]). Let $W$ be a symplectic space with Poisson tensor $\Lambda$ and consider an action by $\mathfrak{s o}_{3}$, generated by Hamiltonian vector fields $\Lambda\left(d L_{i}\right), i=1 \ldots 3$ where $L_{1}, L_{2}, L_{3} \in \mathcal{C}^{\infty}(W)$ satisfy the following Poisson bracket relations,

$$
\left\{L_{i}, L_{j}\right\}=\epsilon_{i j k} L_{k} .
$$

The Casimir element

$$
Q:=\sum_{i=1}^{3} L_{i}^{2} \in \mathcal{C}^{\infty}(W)
$$

is an invariant of the adjoint action,

$$
\left\{L_{i}, Q\right\}=0, \quad i=1 \ldots 3 .
$$

The Hamiltonian vector fields leave invariant each surface $\{Q=k\}(k \in \mathbb{C})$, and each such surface is a symplectic leaf with an induced Poisson structure.

The problem is to invent an equivariant ordering (an invariant star-product $*$ ) such that the star-polynomial $\mathcal{W}(Q):=\sum_{i=1}^{3} L_{i} * L_{i}$ is central,

$$
L_{i} * \mathcal{W}(Q)=\mathcal{W}(Q) * L_{i},
$$

and fixed,

$$
\exists q \in \mathbb{C} ; \quad \forall f \in \mathcal{C}^{\infty}(W), \mathcal{W}(Q) * f=q f .
$$

\subsection{DEFINITION OF $\mathfrak{g}$-INVARIANT STAR-PRODUCTS}

Let $G$ be a Lie group, $G_{e}$ the connected component of $G$ containing the neutral element, $\mathfrak{g}$ the Lie algebra of $G$, and $\mathfrak{g}^{\prime}$ the dual vector space. Given an orbit $W$ of the coadjoint action of $G_{e}$ in $\mathfrak{g}^{\prime}$ we have a homomorphism from the symmetric algebra $\mathrm{S}(\mathfrak{g})$ into $\mathcal{C}^{\infty}(W)$. We identify $\mathfrak{g}$ with $\mathfrak{g}^{\prime}$ as a vector space. Then there is a natural Poisson structure $\{\cdot, \cdot\}$ on $W$ (Berezin-Kirillov-Kostant-Souriau Poisson structure scaled by $\hbar$ ), such that

$$
\forall a, b \in \mathfrak{g}, \hbar\{a, b\}=[a, b] .
$$

DEFINITION 4.1. A star-product on a coadjoint orbit $W$ is $\mathfrak{g}$-invariant if, for all $k \in \mathbb{C}, a, b \in \mathfrak{g}, f, g \in \mathcal{C}^{\infty}(W)$, we have the three following properties

(1) $k * a=a * k=k a$,

(2) $a * b-b * a=\hbar\{a, b\}$,

(3) $\{a, f * g\}=\{a, f\} * g+f *\{a, g\}$. 
Throughout, we are interested in algebraic star-products, star-products that are defined (only) on $\mathrm{S}(\mathfrak{g})$, the set of polynomial functions on $\mathfrak{g}^{\prime}$.

Remark 4.2. Coadjoint orbits provide a plethora of symplectic manifolds, but to invoke the assistance of a Lie group for that purpose alone is somewhat odd. It seems more natural, in this context, to investigate star-products that incorporate additional elements of group theoretical structure.

Given an associative star-product $*$ on $W$, a linear map $\mathcal{W}$ from the symmetric algebra into $\mathcal{C}^{\infty}(W)[[\hbar]]$ is defined as follows,

$$
\mathrm{S}(\mathfrak{g}) \rightarrow \mathcal{C}^{\infty}(W)[[\hbar]], \quad a^{n} \mapsto \mathcal{W}\left(a^{n}\right):=(a *)^{n}, \quad a \in \mathfrak{g} .
$$

For any polynomial $P(a)$ we write $P(a, *)$ for $\mathcal{W}(P(a))$. Conversely, any invertible linear map $\mathcal{W}$ that associates an element of $\mathcal{C}^{\infty}(W)$ to each symmetrized formal star monomial defines an associative star-product on $\mathcal{C}^{\infty}(W)$.

The original Moyal product is the unique associative, invariant product for $W=$ $\mathbb{R}^{2 N}$ with the standard Poisson bracket such that $\mathcal{W}\left(a^{n}\right)=a^{n}$ for every $a$ that is linear in the natural coordinates. It is invariant under the Lie algebra of affine symplectic transformations. The domain includes the space of regular functions (the space of polynomials in the generators).

A recipe for the construction of all invariant star-products for any compact, semi-simple Lie algebra, on any regular coadjoint orbit, was formulated 30 years ago (see [5,29]). For non regular orbits of a compact group see [60].

DEFINITION 4.3. A star-product on $W$ is non degenerate if the space of starpolynomials (actually, the image by $\mathcal{W}$ ) is dense in the space $\mathcal{C}^{\infty}(W)$.

DEFINITION 4.4. ([53, p. 185], [54, p. 368]) Let $\pi$ be an irreducible representation of the universal enveloping algebra $U(\mathfrak{g})$, and let $Z(U(\mathfrak{g}))$ be the center of $U(\mathfrak{g})$. According to Schur's lemma, for every $X \in \mathfrak{g}$, we have $\pi(X)=\lambda_{\pi}(X) \mathbf{1}$, and the map

$$
Z(U(\mathfrak{g})) \rightarrow \mathbb{C}, \quad X \mapsto \lambda_{\pi}(X)
$$

is a morphism of algebras that is called the infinitesimal character of $\pi$.

DEFINITION 4.5. [54, p. 155] A regular (or generic) coadjoint orbit is a coadjoint orbit of maximal dimension (we will see in Section 5.1 that there exists a unique regular nilpotent orbit). 


\subsection{EXAMPLES RELATED TO $\mathfrak{s o}_{n}$}

The following theorem describes star-products in the case of a regular coadjoint orbit for $\mathfrak{s o}_{n}$.

THEOREM 4.6. ([5] $§ 11$ and [6])

- Let $\mathfrak{g}:=\mathfrak{s o}_{n}$. Let $P_{0}:=1, P_{1}(a):=a,\left\{P_{2}, P_{3}, \ldots\right\}$ be a complete set of homogeneous, irreducible, harmonic elements of the projection of $\mathrm{S}\left(\mathfrak{g}^{\prime}\right)$ on a regular coadjoint orbit $W$, and $\left\{P_{n}(a, *) / n \in \mathbb{N}\right\}$ the corresponding set of symmetric star-polynomials. Then an associative, non degenerate, invariant star-product on $W$ is given by an infinitesimal character $Z(\mathrm{U}(\mathfrak{g})) \rightarrow \mathbb{C}$ and by the formulas

$$
\begin{aligned}
& P_{n}(a, *):=C_{n} P_{n}(a), C_{n} \in \mathbb{C}-\{0\}, \quad C_{0}=C_{1}=1, \\
& a * b-b * a=\hbar[a, b], \quad a, b \in g, \quad n=0,1 .
\end{aligned}
$$

- In the case when $\mathfrak{g}=\mathfrak{s o}_{3}, P_{n}(a)$ is a solid Legendre polynomial and the polynomials $P_{n}(a, *)$ can be obtained from the recursion relation (found and solved in [6])

$$
(n+1) P_{n+1}(a, *)=(2 n+1) a * P_{n}(a, *)-n\left(q+\frac{1-n^{2}}{4} \hbar^{2}\right)|a|^{2} P_{n-1}(a, *),
$$

with $P_{0}(a, *)=1$. The parameter $q$ is the image of the Casimir operator $\sum_{i=1}^{3} L_{i} * L_{i}$ by the infinitesimal character.

\section{Some finite-dimensional representations of $\mathfrak{g}$}

An invariant star-product gives an action of $\mathfrak{g}$ on the star algebra, and on $\mathcal{C}^{\infty}(W)$, by the homomorphisms $\pi_{l}: a \mapsto a *$ and similarly by $\pi_{r}: a \mapsto *(-a)$, defined by

$$
\pi_{l}(a) f=a * f \quad \text { and } \quad \pi_{r}(a) f=-f * a, \forall f \in \mathcal{C}^{\infty}(W) .
$$

Questions of domains of these left and right multiplicative operations have not yet been adequately discussed, to our knowledge.

In the case of compact Lie algebras we expect to find finite dimensional representations. How this actually comes about can be seen explicitly in the case of $\mathfrak{g}=\mathfrak{s o}_{3}$.

PROPOSITION 4.7. Let $\mathfrak{g}=\mathfrak{s o}_{3}$. An invariant, associative star-product is of one of two types, both defined as in Equation (10).

(1) All $C_{n} \neq 0$; the action $\pi_{l}$ or $\pi_{r}$ generated by $a *$ or $*(-a)$, a $\in \mathfrak{g}$ is not semisimple. In this case the choice $C_{n}=1+o(\hbar)$ for $n>1$ provides an equivariant deformation for every value $q$ of the Casimir $\sum_{i=1}^{3} L_{i} * L_{i}$ : we have a one-parameter family of star-products. 
(2) If the image of the Casimir by the infinitesimal character takes the value $q=$ $l(l+1)$, with $2 l \in \mathbb{N}$, then the algebra of star polynomials contains an ideal generated by $P_{2 l+1}(a, *)$. The quotient is a finite dimensional *-algebra and is spanned by the projection of $\left\{P_{n}(a, *) / n=0 \ldots 2 l\right\}$. The action of $a *$ and $*(-a)$ is equivalent to the direct product of two copies of the irreducible representation of $\mathfrak{s u}_{2}$, each with dimension $2 l+1$. The polynomial $P_{2 l+1}(a, *)$ reduces in this case to

$$
P_{2 l+1}(a, *) \propto \prod_{m=-l}^{l}(a *-m|a|), \quad a=\sum_{i=1}^{3} a_{i} L_{i}, \quad|a|:=\sqrt{\sum_{i=1}^{3} a_{i}^{2}},
$$

and every $P_{n}(a, *)$ with $n>2 l+1$ contains $P_{2 l+1}(a, *)$ as a factor.

The spectrum, the space of maximal ideals, has a finite number of disconnected components.

\section{Minimal Orbits, Representations, and Joseph Ideals}

\subsection{ADJOINT AND COADJOINT ORBITS}

In this section, we recall some results about adjoint and coadjoint orbits. See [17] for more details (and [54] for examples and other properties of coadjoint orbits). Let $\mathfrak{g}$ be a complex semi-simple Lie algebra, and $G$ a connected semi-simple complex Lie group with Lie algebra $\mathfrak{g}$.

DEFINITION 5.1. - Let $X \in \mathfrak{g}$. We say that $X$ is semi-simple (resp. nilpotent) if $X$ is a semi-simple (resp. nilpotent) endomorphism of $\mathfrak{g}$. The adjoint orbit of $X$ is the set $\mathcal{O}_{X}:=\left\{A d_{g}(X) ; g \in G\right\}$. This is said to be semi-simple (resp. nilpotent) if $X$ is semi-simple (resp. nilpotent).

- Let $\varphi \in \mathfrak{g}^{\prime}$ and set $\mathfrak{g}^{\varphi}:=\left\{X \in \mathfrak{g} / a d_{X}^{*} \varphi=0\right\}$. It is the Lie algebra of the isotropy group of $\varphi$ for the coadjoint action. We say that $\varphi$ is semi-simple (resp. nilpotent) if $\mathfrak{g}^{\varphi}$ is reductive in $\mathfrak{g}$ (resp. $\left.\varphi\right|_{\left.\mathfrak{g}^{\varphi}=0\right)}$ ). We denote by $\mathcal{S}$ (resp. $\mathcal{N}$ ) the set of semi-simple (resp. nilpotent) elements of $\mathfrak{g}^{\prime}$. The coadjoint orbit of $\varphi$ is the set $\mathcal{O}_{\varphi}:=\left\{A_{g}^{*}(\varphi) ; g \in G\right\}$. It is said to be semi-simple (resp. nilpotent) if $\varphi$ is semi-simple (resp. nilpotent).

For $\varphi \in \mathfrak{g}^{\prime}$, set

$$
\omega_{\varphi}: \mathfrak{g} \times \mathfrak{g} \rightarrow \mathbb{C}, \quad(X, Y) \mapsto \varphi([X, Y])
$$

Then $\omega_{\varphi}$ is a symplectic form on $\mathfrak{g} / \mathfrak{g}^{\varphi} \simeq T_{\varphi} \mathcal{O}_{\varphi}$, and $\omega_{\varphi}$ extends to a complex symplectic structure on $\mathcal{O}_{\varphi}$. See for example [64] for a proof of this result. 
Since $\mathfrak{g}$ is semi-simple, the Killing form $\kappa$ is non degenerate. Hence the isomorphism of $\mathfrak{g}$-spaces

$$
i_{\kappa}: \mathfrak{g} \rightarrow \mathfrak{g}^{\prime}, \quad X \mapsto(Y \mapsto \kappa(X, Y)) .
$$

Then we have, because of the invariance of $\kappa$,

$$
i_{\kappa}\left(\mathcal{O}_{X}\right)=\mathcal{O}_{i_{\kappa}(X)} .
$$

Therefore there exists a bijective correspondence between the adjoint and the coadjoint orbits that preserves the properties of semi-simplicity and nilpotency.

In the sequel, we will sometimes identify adjoint and coadjoint orbits.

Let $\mathfrak{h}$ be a Cartan subalgebra of $\mathfrak{g}$, and $W$ its Weyl group. Then we have the following classification of semi-simple orbits.

THEOREM 5.2. The set of semi-simple orbits is in bijective correspondence with the set $\mathfrak{h} / W$. Thus this set is infinite.

On the contrary, the set $\mathcal{N} \mathcal{I} \mathcal{L P}$ of nilpotent orbits is finite. It is in bijection with the set of weighted Dynkin diagrams of $\mathfrak{g}$. The classification results from theorems of Jacobson-Morozov, Kostant, and Mal'cev. See [17] for these theorems and their proof. Moreover, there exists a partial order on the set $\mathcal{N} \mathcal{I} \mathcal{L}$, defined by

$$
\forall \mathcal{O}, \mathcal{O}^{\prime} \in \mathcal{N} \mathcal{I} \mathcal{L P}, \quad\left(\mathcal{O} \preceq \mathcal{O}^{\prime} \Leftrightarrow \overline{\mathcal{O}} \subseteq \overline{\mathcal{O}^{\prime}}\right)
$$

where $\overline{\mathcal{O}}$ is the Zariski closure of $\mathcal{O}$.

The following theorems define the three canonical nilpotent orbits, the principal, the subregular, and the minimal orbits. We recall that the aim of this article is to study star-products on the minimal orbit. Here, $\mathfrak{g}$ is again assumed to be a complex semi-simple Lie algebra.

THEOREM 5.3. (Siebenthal, Dynkin, Kostant) There exists a unique nilpotent orbit of maximal dimension, equal to $\operatorname{dim} \mathfrak{g}-\operatorname{rank} \mathfrak{g}$. This orbit, denoted by $\mathcal{O}_{\text {princ }}$, is called the principal (or regular) orbit and is dense in $\mathcal{N}$.

THEOREM 5.4. (Steinberg) There exists a unique nilpotent orbit of dimension $\operatorname{dim} \mathfrak{g}-\operatorname{rank} \mathfrak{g}-2$. This orbit, denoted by $\mathcal{O}_{\text {subreg, }}$, is called the subregular orbit and is dense in $\mathcal{N} \backslash \mathcal{O}_{\text {princ. }}$

THEOREM 5.5. There exists a unique nonzero orbit of minimal dimension. This orbit, denoted by $\mathcal{O}_{\mathrm{min}}$, is called the minimal orbit and is contained in the closure of any nilpotent orbit. We have $\overline{\mathcal{O}_{\min }}=\mathcal{O}_{\min } \sqcup\{0\}$. 


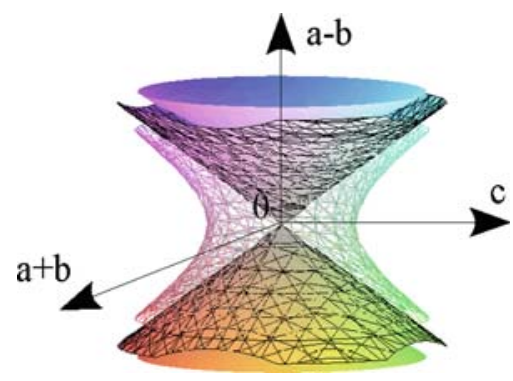

Figure 1. Coadjoint orbits of $\mathfrak{s o}(2,1)$.

EXAMPLE 5.6. - The smallest $n \in \mathbb{N}^{*}$ for which the three canonical nilpotent orbits of $\mathfrak{s l}_{n}$ are distinct is $n=4$. In this case, the nilpotent orbits of $\mathfrak{s l}_{4}$ are given in the following table.

Orbit $\quad \mathcal{O}_{[4]}=\mathcal{O}_{\text {princ }} \mathcal{O}_{[3,1]}=\mathcal{O}_{\text {subreg }} \mathcal{O}_{[2,2]} \quad \mathcal{O}_{[2,1,1]}=\mathcal{O}_{\min } \mathcal{O}_{[1,1,1,1]}=\{0\}$

Dimension $12 \quad 10 \quad 8 \quad 6 \quad 0$

- Consider $\mathfrak{s l}_{2}=\left\{\left(\begin{array}{cc}a & b \\ c & -a\end{array}\right) /(a, b, c) \in \mathbb{C}^{3}\right\}$.

Then the semi-simple orbits are the sets $\{0\}$ and $\left\{(a, b, c) \in \mathbb{C}^{3} / a^{2}+b c=k\right\}$, for $k \in \mathbb{C}^{*}$.

$$
\mathcal{O}_{\text {princ }}=\mathcal{O}_{\text {subreg }}=\mathcal{O}_{\text {min }}=\left\{(a, b, c) \in \mathbb{C}^{3} / a^{2}+b c=0\right\} \backslash\{0\}
$$

(cone without 0). For the convenience of the reader we represent here in Figure 1 the orbits in $\mathbb{R}^{3}$, i.e. the coadjoint orbits of the real Lie algebra $\mathfrak{s o}(2,1)$ considered in Section 5.3.

\subsection{NILPOTENT ORBITS AND JOSEPH IDEALS}

Let $\mathfrak{g}$ be a complex finite dimensional semi-simple Lie algebra, $V$ a finite dimensional vector space, and $\pi: \mathfrak{g} \rightarrow \mathfrak{g l}(V)$ an irreducible representation of $\mathfrak{g}$. This induces a unique representation $\widehat{\pi}$ of the universal enveloping algebra $U(\mathfrak{g})$ of $\mathfrak{g}$. The two-sided ideal $I:=\operatorname{Ker} \widehat{\pi}$ is called the primitive ideal associated with $\pi$.

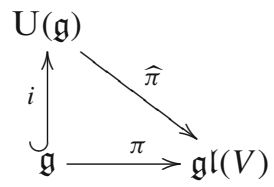


According to the Poincaré-Birkhoff-Witt theorem [18], there is an isomorphism of graded algebras $\operatorname{Gr}(U(\mathfrak{g})) \simeq S(\mathfrak{g})$, where $S(\mathfrak{g})$ is the symmetric algebra of $\mathfrak{g}$, the algebra of polynomial functions on $\mathfrak{g}^{\prime}$. And $J:=\operatorname{Gr}(\operatorname{Ker} \widehat{\pi})$ is a graded ideal of $\mathrm{S}(\mathfrak{g})$. Let $\mathcal{V}(I)$ be the variety of common zeros of $J$ in $\mathfrak{g}^{\prime}$.

The two following theorems link the primitive ideals with the nilpotent orbits and allow us to define the Joseph ideal associated with the minimal orbit.

THEOREM 5.7. (Borho, Brylinski, Joseph [11,52]) Let I be a primitive ideal of $\mathrm{U}(\mathfrak{g})$. Then $\mathcal{V}(I)$ is the closure $\overline{\mathcal{O}}$ of a nilpotent orbit $\mathcal{O}$ of $\mathfrak{g}$.

But every coadjoint nilpotent orbit is determined by its closure, therefore we may say that $\mathcal{O}$ is the nilpotent orbit associated with the primitive ideal $I$.

THEOREM 5.8. (Joseph [49,50]) Let $\mathfrak{g}$ be a complex simple Lie algebra, and $\mathcal{O}_{\min }$ its minimal orbit. Then there exists a unique completely prime two-sided ideal $J_{\min }$ in $\mathrm{U}(\mathfrak{g})$ such that $\mathcal{V}\left(J_{\min }\right)=\mathcal{O}_{\min } \sqcup\{0\}$. This ideal is primitive and called the Joseph ideal.

The root space of the highest root of $\mathfrak{g}$ is contained in the minimal orbit. More precisely, let $\beta$ be the highest root of $\mathfrak{g}$ (see $[12,48]$ ), and $\mathfrak{g}_{\beta}$ the associated root space,

$$
\mathfrak{g}_{\beta}:=\{X \in \mathfrak{g} ; \quad \forall h \in \mathfrak{h}, \quad[h, X]=\beta(h) X\} .
$$

Then we have $\mathfrak{g}_{\beta} \subset i_{\kappa}^{-1}\left(\mathcal{O}_{\min }\right)$ (see [50]).

The following result, due to Kostant [58], is an important tool for our study of minimal orbits.

THEOREM 5.9. (Kostant) The closure of the minimal orbit $\left(\mathcal{O}_{\min } \sqcup\{0\}\right)$ is an algebraic variety

$$
\overline{\mathcal{O}_{\min }}=\left\{\left(x_{1}, \ldots, x_{N}\right) \in \mathbb{C}^{N} ; \forall r \in R, r\left(x_{1}, \ldots, x_{N}\right)=0\right\},
$$

where $N$ is the dimension of $\mathfrak{g}$ and $R:=\left\{g_{1}, \ldots, g_{K}\right\}$ is a finite set of quadratic relations.

The set of regular functions on $\overline{\mathcal{O}_{\min }}$ is the set

$$
\mathcal{F}\left(\overline{\mathcal{O}_{\min }}\right) \simeq \mathbb{C}\left[x_{1}, \ldots, x_{N}\right] /\langle R\rangle .
$$

In the sequel, we will often call $\overline{\mathcal{O}_{\min }}$ the minimal orbit. Similarly, we will often talk about relations defining the minimal orbit, even if these relations define of course the closure of the orbit.

The last theorem of this section links the primitive ideals to the highest weight modules. See [19] and [51]. 
THEOREM 5.10. (Duflo, Joseph) Every primitive ideal of $\mathrm{U}(\mathfrak{g})$ is the annihilator of a simple highest weight module.

\subsection{COADJOINT ORBITS OF $\mathfrak{s o}(2,1)$}

Consider the real Lie algebra $\mathfrak{s o}(2,1)$, and let $\left(L_{1}, L_{2}, L_{3}\right)$ be its standard basis, with relations

$$
\left[L_{i}, L_{j}\right]=\epsilon_{i j k} L_{k}
$$

and Killing form $\kappa$ such that, setting $L:=a_{1} L_{1}+a_{2} L_{2}+a_{3} L_{3}$, we have

$$
\kappa(L, L)=g^{i j} a_{i} a_{j}=-\left(a_{1}\right)^{2}-\left(a_{2}\right)^{2}+\left(a_{3}\right)^{2} .
$$

The moment map interprets $L_{1}, L_{2}, L_{3}$ as coordinates for the coadjoint space; in this role we write them $\mathbf{x}:=\left(x_{1}, x_{2}, x_{3}\right)$. Set $g(\mathbf{x}):=g^{i j} x_{i} x_{j}$. Then the semi-simple orbits are the (real) algebraic varieties

$$
\left\{\mathbf{x} \in \mathbb{R}^{3} ; g(\mathbf{x})=k\right\},
$$

for $k \neq 0$, and the closure of the minimal nilpotent orbit is the (real) algebraic variety

$$
\overline{\mathcal{O}_{\min }}=\left\{\mathbf{x} \in \mathbb{R}^{3} ; g(\mathbf{x})=0\right\} .
$$

This variety is a simple cone in the sense of Section 3.2.

The regular functions on $\overline{\mathcal{O}_{\min }}$ are the restrictions to $\overline{\mathcal{O}_{\min }}$ of polynomials in $x_{1}, x_{2}, x_{3}$. The set of regular functions is isomorphic to

$$
\mathbb{R}\left[x_{1}, x_{2}, x_{3}\right] /\langle g\rangle .
$$

The first order star-product $x_{i} * x_{j}=x_{i} x_{j}+\hbar C_{1}\left(x_{i}, x_{j}\right)$ is equivariant if $C_{1}^{-}\left(x_{i}, x_{j}\right)=\frac{1}{2} \epsilon_{i j k} x_{k}$ and $C_{1}^{+}\left(x_{i}, x_{j}\right)$ is proportional to $g_{i j}$. Since $\kappa$ is non degenerate, there is a two parameter family of essential first order equivariant deformations, indexed by $\hbar$ and $\left.C_{1}^{+}\right|_{x=0}$. Every first order equivariant deformation can be extended, for example by the method outlined above, to an invariant star-product.

\subsection{MINIMAL ORBIT OF $\mathfrak{s l}_{n}$}

The minimal orbit is defined by the relations

$$
U_{a}^{b} U_{c}^{d}-U_{a}^{d} U_{c}^{b}=0, \forall a, b, c, d=1 \ldots n .
$$

We also have the relation $\sum_{j=1}^{n} U_{j}^{j}=0$ (trace zero).

Note that if a matrix $U \in \mathfrak{s l}_{n}$ belongs to the minimal orbit, then $U^{2}=0$. 
Remark 5.11. The relations (11) are solved by the factorization

$$
U_{a}^{b}=p_{a} q^{b}, \text { with } q \cdot p:=q^{a} p_{a}=0,
$$

which defines an embedding of $\mathfrak{g}$ into the space of second order polynomials on $P^{2 n-2}:=\mathbb{R}^{2 n} /(p \cdot q, \approx)$, where $\approx$ is the equivalence relation defined by

$$
\forall \lambda>0, \quad\left(\lambda p, \lambda^{-1} q\right) \approx(p, q) .
$$

A star-product can be defined on this space by introducing the Poisson bracket defined by

$$
\left\{q^{b}, p_{a}\right\}=\delta_{a}^{b},\left\{q^{b}, q_{d}\right\}=\left\{p^{a}, p_{c}\right\}=0,
$$

and quantizing this in the manner of Weyl. See Sections 5.6 and 6.2.

\subsection{MINIMAL ORBITS OF $\mathfrak{s p}_{2 n}, \mathfrak{s o}_{n}$ AND $\mathfrak{g}_{2}$}

The Lie algebra $\mathfrak{s p}_{2 n}$ is the algebra of traceless matrices that leave invariant a skew-symmetric, non degenerate 2-form $\eta, U_{a}^{b} \eta_{b c}=: L_{a c}=L_{c a}$.

We choose the embedding $L_{a c}=\xi_{a} \xi_{c}$, with $\xi_{1}, \ldots, \xi_{2 n}=q^{1}, \ldots, q^{n}, p_{1}, \ldots, p_{n}$, which incorporates all the relations that define the minimal orbit. In fact, consider the polynomial algebra $A:=\mathbb{C}\left[q^{1}, \ldots, q^{n}, p_{1}, \ldots, p_{n}\right]$, endowed with the standard Poisson bracket

$$
\{F, G\}:=\nabla_{p} F \cdot \nabla_{q} G-\nabla_{q} F \cdot \nabla_{p} G .
$$

The subspace $A(2)$ of the elements of degree 2 is a Lie algebra for $\{\cdot, \cdot\}$ that is isomorphic to the Lie algebra $\mathfrak{s p}_{2 n}$. The relations that define the minimal orbit are

$$
L_{a b} L_{c d}=L_{a d} L_{c b}, \forall a, b, c, d=1 \ldots n \text {. }
$$

The Lie algebra $\mathfrak{s o}_{n}$ is the algebra of traceless matrices that leave invariant a symmetric, non degenerate 2 -form $\eta$. Analogy with $\mathfrak{s p}_{2 n}$ suggests using Grassmann variables, replacing the commutative affine algebra by a super-commutative super Lie algebra, as is done in field theories with fermions. More precisely, we may replace the polynomial algebra $A=S\left(E^{\prime}\right)$ (where $E=\operatorname{Vect}\left(q^{1}, \ldots, q^{n}, p_{1}, \ldots, p_{n}\right)$ ) given for $\mathfrak{s p}_{2 n}$ by the algebra $\Lambda^{*}\left(E^{\prime}\right)$, and the Poisson bracket $\{\cdot, \cdot\}$ by a convenient Poisson super-bracket. Then the subspace $\Lambda^{2}\left(E^{\prime}\right)$ is a Lie algebra isomorphic to the Lie algebra of the orthogonal group. For more information on these spaces and on the link with the orthosymplectic group, see [41, Chapter 9]. An alternative is the embedding $L_{a c}=q_{a} p_{c}-q_{c} p_{a}$ (with $q_{a}=q^{b} \eta_{b a}$ ). The relations that define the minimal orbit are

$$
\sum_{\operatorname{cycl}(a b c)} L_{a b} L_{c d}=0, \quad \forall d=1 \ldots n, \quad \sum_{b, c=1 \ldots n} \eta^{b c} L_{a b} L_{c d}=0, \quad \forall a, d=1 \ldots n,
$$


where $\eta^{a b}$ is the general term of the inverse of the matrix $\left(\eta_{a b}\right)_{(a, b) \in\{1, \ldots, n\}}$. The first relation is implied by the embedding and the second can be incorporated by restriction to $\eta^{a b} p_{a} p_{b}=\eta^{a b} q_{a} q_{b}=q^{a} p_{a}=0$ and projecting on a quotient.

The Lie algebra $\mathfrak{g}_{2}$ is isomorphic to the Lie algebra of derivations of the non associative algebra $\mathbb{O}$ of complex Cayley octonions. This is a subalgebra of $\mathfrak{s o}_{7}$ (see $[34,48,65])$. The minimal orbit can be parametrized as that of $\mathfrak{s o}_{7}$ with the additional condition $p \times q=0$.

\subsection{ASSOCIATED REPRESENTATIONS AND JOSEPH IDEALS}

5.6.1. Background The ideals in the universal enveloping algebra of a compact simple Lie algebra are fixed by a central character. The non compact case is more interesting. An example is well known to physicists. This is the real Lie algebra $\mathfrak{s o}(4,2)$, with the usual basis $\left\{L_{a b} ; 1 \leq a<b \leq 6\right\}$ and relations

$$
\left[L_{a b}, L_{c d}\right]=\eta_{b c} L_{a d}-\eta_{a c} L_{b d}-\eta_{b d} L_{a c}+\eta_{a d} L_{b c},
$$

where $\eta$ is the pseudo Euclidean metric. The tensor $g^{b c}\left(L_{a b} L_{c d}+L_{d b} L_{c a}\right)$ in the universal enveloping algebra reduces, in a certain irreducible and unitarizable representation, to fixed numerical values, so that the relations

$$
\eta^{b c}\left(L_{a b} L_{c d}+L_{d b} L_{c a}\right)=-\hbar^{2} \eta_{a d}
$$

hold in the representation. This particular representation appears in the analysis of the Schrödinger theory of the hydrogen atom and in conformal field theory. The algebra also enters the description of Keplerian orbits on a six-dimensional phase space.

The orbit is all the more interesting since Kostant's method of geometric quantization encounters a difficulty: the non existence of an invariant polarization [59]. It was shown by Joseph that this is true for all minimal orbits except the case of $\mathfrak{s l}_{n}$ [50]. Although the corresponding quantum theory is known, an invariant WignerWeyl correspondence [5] is not. We know that an invariant star-product associated with such a correspondence exists, but it has not been constructed. Nevertheless, relation (15) suggests that there is an invariant star-product such that

$$
\eta^{b c}\left(L_{a b} * L_{c d}+L_{d b} * L_{c a}\right)=-\hbar^{2} \eta_{a d} .
$$

A question that motivated this work is whether such a deformation exists, and if it is a deformation in the direction of the Poisson bracket,

$$
f * g=f g+\frac{\hbar}{2}\{f, g\}+\sum_{j=2}^{\infty} C_{j}(f, g) \hbar^{j} .
$$

The undoubted presence of interesting homology on the (closure of) this orbit was expected to play a role in invariant quantization. A preliminary exploration of the associated representations will show us what to expect. 
5.6.2. Associated representations of $\mathfrak{s l}_{n}$ Let $V_{N}$ denote the vector space of polynomial functions on $\mathbb{C}^{n}$ spanned by the set of functions $x_{1}^{r_{1}}, \ldots, x_{n}^{r_{n}}$, with $r_{j} \in \mathbb{N}$, and $N=\sum_{i=1}^{n} r_{i}$ fixed. For $a, b=1 \ldots n$, let $\widetilde{U}_{a}^{b}$ be the operator in $V_{N}$, given by

$$
\widetilde{U}_{a}^{b}=\hbar\left(x_{a} \partial_{b}-\frac{N}{n} \delta_{a}^{b}\right),
$$

where $\partial_{b}$ stands for the partial derivation with respect to $x_{b}$. Then $\left[\widetilde{U}_{a}^{b}, \widetilde{U}_{c}^{d}\right]=$ $\hbar\left(\delta_{c}^{b} \widetilde{U}_{a}^{d}-\delta_{a}^{d} \widetilde{U}_{c}^{b}\right)$, and the map

$$
\mathfrak{s l}_{n} \rightarrow L\left(V_{N}\right), \quad E_{a b} \mapsto \frac{1}{\hbar} \widetilde{U}_{a}^{b} \quad \text { if } a \neq b, \quad E_{a a}-\frac{1}{n} I_{n} \mapsto \frac{1}{\hbar} \widetilde{U}_{a}^{a}
$$

is a morphism of Lie algebras, a representation of $\mathfrak{s l}_{n}$.

To be more precise, consider the real form $\mathfrak{s u}(n-1,1)$, with the compact subalgebra $\mathfrak{s u}_{n-1}$ generated by $\left\{\widetilde{U}_{a}^{b} / a, b=1 \ldots n-1\right\}$. Taking $r_{1}, \ldots, r_{n-1}$ to run over the natural numbers we obtain, for a range of values of $N$, a unitarizable highest weight representation, with the highest weight reducing to zero on $\mathfrak{s u}_{n-1}$.

The relations that define the minimal orbit are $U_{a}^{b} U_{c}^{d}-U_{a}^{d} U_{c}^{b}=0$, while a direct calculation gives ${ }^{5}$

$$
\widetilde{U}_{a}^{b} \widetilde{U}_{c}^{d}-\widetilde{U}_{a}^{d} \widetilde{U}_{c}^{b}=\hbar \delta_{c}^{b} \widetilde{U}_{a}^{d}-\hbar \frac{N}{n}\left(\delta_{a}^{b} \widetilde{U}_{a}^{b} \widetilde{U}_{c}^{d}+\delta_{c}^{d} \widetilde{U}_{a}^{b}\right)-\hbar^{2} \frac{N}{n}\left(\frac{N}{n}+1\right) \delta_{a}^{b} \delta_{c}^{d}-(b \leftrightarrow d) .
$$

An invariant deformation in the direction of the Poisson bracket would have the following form

$$
U_{a}^{b} * U_{c}^{d}=U_{a}^{b} U_{c}^{d}+\frac{\hbar}{2}\left\{U_{a}^{b}, U_{c}^{d}\right\}+\hbar^{2} C^{+}\left(U_{a}^{b}, U_{c}^{d}\right) .
$$

In this setting, because of the very strong relations that characterize the orbit, the most general equivariant, symmetric 2 -form $C^{+}$takes the form

$$
\hbar C^{+}\left(U_{a}^{b}, U_{c}^{d}\right)=\frac{k}{2}\left(\delta_{c}^{b} U_{a}^{d}+\delta_{a}^{d} U_{c}^{b}-\frac{2}{n}\left(\delta_{a}^{b} U_{c}^{d}+\delta_{c}^{d} U_{a}^{b}\right)\right)+k^{\prime}\left(\delta_{a}^{d} \delta_{c}^{b}-\frac{1}{n} \delta_{a}^{b} \delta_{c}^{d}\right) .
$$

These relations have the same form as Equation (17) if the parameters $k, k^{\prime}$ are appropriately related to the degree $N$ of the homogeneous functions in the vector space $V_{N}$, and if we have

$$
k\left(1+\frac{2}{n}\right)=-\hbar\left(1+2 \frac{N}{n}\right), \quad k^{\prime}\left(1+\frac{1}{n}\right)=\hbar^{2} \frac{N}{n}\left(\frac{N}{n}+1\right) .
$$

It turns out that such a deformation exists if and only if the parameters $k$ and $k^{\prime}$ are related to each other, precisely as implied by Equation (20).

${ }^{5}$ The notation $\leftrightarrow$ has the following meaning: "Formula $(a, b)+(a \leftrightarrow b)$ " means "Formula $(a, b)+$ Formula $(b, a)$ ". Similarly, "Formula $(a, b, c, d)-(a \leftrightarrow b)-(c \leftrightarrow d)+(a \leftrightarrow b, c \leftrightarrow d)$ " means "Formula $(a, b, c, d)-\operatorname{Formula}(b, a, c, d)-\operatorname{Formula}(a, b, d, c)+\operatorname{Formula}(b, a, d, c) "$. 
5.6.3. Associated representations of $\mathfrak{s o}_{n}$ and $\mathfrak{s p}_{2 n}$

\section{Study of $\mathfrak{s o}_{n}$}

Let $V_{N}=\left\langle x_{1}^{r_{1}}, \ldots, x_{n}^{r_{n}}\right\rangle_{\mathbb{C}}$ be the space defined in Section 5.6.2, and $\eta$ a symmetric, non degenerate 2 -form on $\mathbb{C}^{n}$. Let $\partial_{a}:=\eta_{a b} \partial_{b}$ and $\widetilde{L}_{a b}:=\hbar\left(x_{a} \partial_{b}-x_{b} \partial_{a}\right)$, which gives a formal representation of $\mathfrak{s o}_{n}$ and the formulae

$$
\widetilde{L}_{a b} \widetilde{L}_{c d}=\hbar^{2}\left(x_{a} x_{c} \partial_{b} \partial_{d}+\eta_{b c} x_{a} \partial_{d}-(a \leftrightarrow b)-(c \leftrightarrow d)+(a \leftrightarrow b, c \leftrightarrow d)\right) .
$$

We want to simplify this representation as much as possible and therefore restrict the variables to the cone $\eta(x, x)=0$ and the space $V_{N}$ to the subspace of harmonic functions. (We verify that the choice $N=2-\frac{n}{2}$ of the degree of homogeneity gives to the Laplace operator a well defined action on functions defined on the cone.) The first term on the right now satisfies the constraints. This is not yet a model for a star-product since the first order operators on the right do not combine to $\widetilde{L}_{a b}$ 's. But another way to write the last relations is

$$
\begin{aligned}
\widetilde{L}_{a b} \widetilde{L}_{c d}= & \hbar^{2}\left(x_{a} x_{c} \partial^{b} \partial^{d}+\frac{1}{2} \eta_{b c}\left(x_{a} \partial^{d}+x_{d} \partial^{a}\right)-(a \leftrightarrow b)-(c \leftrightarrow d)+(a \leftrightarrow b, c \leftrightarrow d)\right)+ \\
& +\frac{\hbar}{2} \eta_{b c}\left(\widetilde{L}_{a d}-(a \leftrightarrow b)-(c \leftrightarrow d)+(a \leftrightarrow b, c \leftrightarrow d)\right) .
\end{aligned}
$$

The first line satisfies the constraints, and if we take this as a model for the classical part, then we are led to look for a star-product of the form

$$
L_{a b} * L_{c d}=L_{a b} L_{c d}+\frac{\hbar}{2} \eta_{b c}\left(L_{a d}-(a \leftrightarrow b)-(c \leftrightarrow d)+(a \leftrightarrow b, c \leftrightarrow d)\right)+o\left(\hbar^{2}\right) .
$$

It turns out that such an invariant star-product exists if and only if the last term is precisely

$$
\hbar^{2}\left(2-\frac{n}{2}\right)(1-n) \kappa\left(L_{a b}, L_{c d}\right),
$$

where $\kappa$ is the Killing form.

See [10] for a complete discussion of singular representations of $\mathfrak{s o}(p, q)$.

EXAMPLE 5.12. In the case $n=6, N=-1$, restricting to the real form $\mathfrak{s o}(4,2)$ and taking $r_{1}, \ldots, r_{4} \in \mathbb{N}, \frac{r_{5}}{r_{6}} \in \mathbb{N}$, we recover the unitarizable representation that is realized on the space of solutions of a massless scalar field in 4 dimensions, in the form discovered by Dirac.

The same representation appears in the theory of the hydrogen atom, where it is realized by self-adjoint operators in $L^{2}\left(\mathbb{R}, \frac{d^{3} r}{r}\right)$.

\section{Study of $\mathfrak{s p}_{2 n}$}

Let $V_{2 n}=\mathbb{C}\left[\xi_{1}, \ldots, \xi_{2 n}\right]$ be the space of polynomials on a complex vector space of dimension $2 n$ endowed with a skew-symmetric, non degenerate 2-form $\eta$ and coordinates $\xi_{1}, \ldots, \xi_{2 n}$. 
A Poisson bracket is defined by $\left\{\xi_{a}, \xi_{b}\right\}=\eta_{a b}$. Let $\partial_{a}:=\eta_{a b} \partial_{b}$ and $\widetilde{L}_{a b}:=$ $\hbar\left(\xi_{a} \partial_{b}+\xi_{b} \partial_{a}\right)$. Then a similar analysis suggests a star-product such that

$$
L_{a b} * L_{c d}=L_{a b} L_{c d}+\frac{\hbar}{2} \eta_{b c}\left(L_{a d}+(a \leftrightarrow b)+(c \leftrightarrow d)+(a \leftrightarrow b, c \leftrightarrow d)\right)+\frac{\hbar^{2}}{2} \eta_{a b} \eta_{c d} .
$$

This is of course the Moyal star-product, restricted to $\mathfrak{s p}_{2 n}$.

\section{6. g-Invariant Star-Products on the Minimal Orbit of a Simple Lie Algebra}

\subsection{COMPUTATION OF THE HOMOLOGY}

Here we rely on Kostant's characterization (Theorem 5.9) of the minimal orbit. We need a generalization of the results of Section 3.2 to this case of multiple relations, up to the level of 3-chains and 3-cochains. It is clear that $\mathrm{HH}_{2}$ is the space spanned by the following chains,

$$
\left(Z_{2,1}\right)_{\alpha}=g_{\alpha}^{i j} x_{i} \otimes x_{j}, \text { for } \alpha \in \mathbb{N}, \quad \text { and } \quad\left(Z_{2,2}\right)_{i j}=x_{i} \wedge x_{j} \text {, for } i, j=1 \ldots N .
$$

Every closed 3-chain is homologous to a linear one,

$$
a=x_{i} \otimes x_{j} \otimes x_{k} A^{i j k},
$$

with $A^{i j k} \in \mathbb{C}$, and this chain is closed if and only if

$$
x_{i} x_{j} A^{i j k}=0=x_{j} x_{k} A^{i j k} .
$$

Hence

$$
A^{i j k}+A^{j i k}=g_{\alpha}^{i j} c_{\alpha}^{k}, \quad A^{i j k}+A^{i k j}=c_{\alpha}^{\prime i} g_{\alpha}^{j k},
$$

with complex coefficients $c_{\alpha}, c_{\alpha}^{\prime}$. This can be solved if and only if

$$
\sum_{\mathrm{cycl}} g_{\alpha}^{i j} c_{\alpha}^{k}=\sum_{\mathrm{cycl}} c_{\alpha}^{\prime i} g_{\alpha}^{j k}
$$

where the sums are over cyclic permutations, which implies that $c_{\alpha}^{\prime}{ }^{i}=c_{\alpha}^{i}+\rho_{\alpha}^{i}$, with the coefficients $\rho_{\alpha}^{i}$ subject to $\sum_{\text {cycl }} \rho_{\alpha}^{i} g_{\alpha}^{j k}=0$, as follows,

$$
6 A^{i j k}=\operatorname{Alt} A^{i j k}+3\left(g_{\alpha}^{i j} c_{\alpha}^{k}-g_{\alpha}^{i k} c_{\alpha}^{j}+g_{\alpha}^{j k} c_{\alpha}^{i}\right)+2\left(\rho_{\alpha}^{i} g_{\alpha}^{j k}-\rho_{\alpha}^{j} g_{\alpha}^{i k}\right), \quad \sum_{\operatorname{cycl}(i j k)} \rho_{\alpha}^{i} g_{\alpha}^{j k}=0 .
$$

The three terms belong respectively to $Z_{3,3}=H H_{3,3}, Z_{3,2}=H H_{3,2}$ (they are of the form listed in Proposition 3.5) and $Z_{3,1}=H_{3,1}$. This third space, empty in the case where there is only one relation, has not been determined. We only have the following conjecture: 
CONJECTURE 6.1. The space $\mathrm{Z}_{3,1}=H_{3,1}$ is spanned by chains of the form

$$
e_{3}(1) g_{\alpha}^{i j} g_{\beta}^{k l}\left(x_{i} \otimes\left\{x_{j}, x_{k}\right\} \otimes x_{l}\right),
$$

where $\left\{g_{\alpha}\right\}$ is the full set of binary relations and $e_{3}(1)$ is the BGS idempotent.

EXAMPLE 6.2. For $\mathfrak{s l}_{n}$, the two relations $U_{a}^{b} U_{c}^{d}-U_{c}^{b} U_{a}^{d}=0, U_{d}^{f} U_{g}^{h}-U_{d}^{h} U_{g}^{f}=0$ generate in this manner the closed chain

$$
U_{a}^{b} \otimes U_{c}^{f} \otimes U_{g}^{h}-U_{c}^{b} \otimes U_{a}^{f} \otimes U_{g}^{h}-U_{a}^{b} \otimes U_{c}^{h} \otimes U_{g}^{f}+U_{c}^{b} \otimes U_{a}^{h} \otimes U_{g}^{f} .
$$

The difficulty will be overcome with the help of the correspondence principle, an adaptation of Weyl's symmetric ordering. It is the subject of the following section.

\subsection{CORRESPONDENCE PRINCIPLE FOR $\mathfrak{g}$-INVARIANT STAR-PRODUCTS}

We take a fresh point of departure. Suppose that an invariant star-product has the following property

$$
S\left(x_{i_{1}} * \cdots * x_{i_{p}}\right)=x_{i_{1}} \ldots x_{i_{p}}+\phi_{i_{1} \ldots i_{p}},
$$

where $S$ stands for the symmetrization, and the function $\phi_{i_{1} \ldots i_{p}}$ belongs to the ideal $\langle\hbar\rangle$ of $\mathbb{C}[[\hbar]]$.

Remark 6.3. We shall postulate that, for each order in $\hbar, \phi_{i_{1} \ldots i_{p}}$ is a polynomial of order less than $p$. In this way we guarantee an important property of the deformation: the Poincaré-Witt basis is preserved. Actually, in the present context this is a weak limitation, since equivariant 2-cochains of higher order are scarce by reason of the constraints.

EXAMPLE 6.4. If $\phi=0$, then we get the quantization rule given by Abellanas and Martinez-Alonso in [1] and called by them Weyl quantization.

Invariance of the star-product requires that the map $\phi$ :

$$
A \rightarrow A, \quad x_{i_{1}} \ldots x_{i_{p}} \mapsto \phi_{i_{1} \ldots i_{p}}
$$

be equivariant for the adjoint action. The only other requirement is the obvious fact that the correspondence must be consistent with the relations that define the variety. Applying these constraints to both sides of Equation (21), we obtain conditions on the map $\phi$. We shall calculate these conditions explicitly for monomials of order 2 and 3. We shall show that these conditions are precisely the same as those implied by associativity, confirming the fact that associativity is not a separate concern. So long as the correspondence is consistent with the constraints 
(now the only issue), detailed knowledge of the restricted homology spaces is not required. Cohomology was crucial for the demonstration of an extension to higher orders (Theorem 3.4), but it is not the best tool for establishing the basis at low orders.

Let the symbol $S$ stand for symmetrization in the order of factors and set

$$
S\left(x_{i} * x_{j}\right)=x_{i} x_{j}+\psi_{i j}, \quad S\left(x_{i} * x_{j} * x_{k}\right)=x_{i} x_{j} x_{k}+\phi_{i j k},
$$

and recall that

$$
x_{i} * x_{j}-x_{j} * x_{i}=\hbar\left\{x_{i}, x_{j}\right\}=\hbar \epsilon_{i j}^{m} x_{m},
$$

The polynomials $\phi_{i j k}$ and $\psi_{i j}$ are assumed to define equivariant maps as explained above.

PROPOSITION 6.5. Assume that the equivariant polynomials $\psi$ and $\phi$ have been chosen so that relations (22) are consistent with the constraints. Then there is an invariant associative star-product such that Equation (22) holds.

Proof. It follows from Equation (22) that

$$
\left(x_{i} x_{j}\right) * x_{k}=x_{i} * x_{j} * x_{k}-\frac{\hbar}{2} \epsilon_{i j}{ }^{m} x_{m} * x_{k}-\psi_{i j} * x_{k} .
$$

The symmetrized star product is

$$
\begin{aligned}
S\left(x_{i} * x_{j} * x_{k}\right) & =\frac{1}{6} \sum_{\sigma \in S_{3}}\left(x_{i} * x_{j} * x_{k}\right)= \\
& =\frac{1}{2} x_{i} * x_{j} * x_{k}+\frac{\hbar}{3} x_{i} *\left\{x_{k}, x_{j}\right\}+\frac{\hbar}{6}\left\{x_{k}, x_{i}\right\} * x_{j}+(i \leftrightarrow j),
\end{aligned}
$$

and we deduce that

$$
\begin{aligned}
\left(x_{i} x_{j}\right) * x_{k}= & x_{i} x_{j} x_{k}+\phi_{i j k}- \\
& -\psi_{i j} * x_{k}-\frac{\hbar}{2}\left(\psi\left(\left\{x_{k}, x_{i}\right\}, x_{j}\right)+(i \leftrightarrow j)\right)- \\
& -\frac{\hbar}{2}\left(\left\{x_{k}, x_{i}\right\} x_{j}+(i \leftrightarrow j)\right)-\frac{\hbar^{2}}{12}\left(\left\{x_{i},\left\{x_{k}, x_{j}\right\}\right\}+(i \leftrightarrow j)\right) .
\end{aligned}
$$

Taking $\psi$ to be equivariant leads to some cancelations,

$$
\begin{aligned}
\left(x_{i} x_{j}\right) * x_{k}= & x_{i} x_{j} x_{k}+\phi_{i j k}-\psi_{i j} x_{k}-\psi\left(\psi_{i j}, x_{k}\right)- \\
& -\frac{\hbar}{2}\left(\left\{x_{k}, x_{i}\right\} x_{j}+(i \leftrightarrow j)\right)-\frac{\hbar^{2}}{12}\left(\left\{x_{i},\left\{x_{k}, x_{j}\right\}\right\}+(i \leftrightarrow j)\right) .
\end{aligned}
$$

Similarly

$$
\begin{aligned}
x_{i} *\left(x_{j} x_{k}\right)= & x_{i} x_{j} x_{k}+\phi_{i j k}-x_{i} \psi_{j k}-\psi\left(x_{i}, \psi_{j k}\right)- \\
& -\frac{\hbar}{2}\left(\left\{x_{k}, x_{i}\right\} x_{j}+(j \leftrightarrow k)\right)-\frac{\hbar^{2}}{12}\left(\left\{\left\{x_{j}, x_{i}\right\}, x_{k}\right\}+(j \leftrightarrow k)\right) .
\end{aligned}
$$


These equations yield explicit expressions for the values of the two-forms $C_{1}$ and $C_{2}$ defined by

$$
\begin{aligned}
& \left(x_{i} x_{j}\right) * x_{k}=x_{i} x_{j} x_{k}+\hbar C_{1}\left(x_{i} x_{j}, x_{k}\right)+\hbar^{2} C_{2}\left(x_{i} x_{j}, x_{k}\right), \\
& x_{i} *\left(x_{j} x_{k}\right)=x_{i} x_{j} x_{k}+\hbar C_{1}\left(x_{i}, x_{j} x_{k}\right)+\hbar^{2} C_{2}\left(x_{i}, x_{j} x_{k}\right) .
\end{aligned}
$$

These values solve the condition for associativity of the star product on linear chains. Theorem 6 then assures us that they can be satisfied in general. The proposition is proved.

In the present approach, associativity is satisfied trivially. What is far from trivial is the existence of a function $\phi_{i j k}$ that solves Equation (24). The obstructions are the constraints. Application of $g_{\alpha}^{i j}$ to the first and $g_{\alpha}^{j k}$ to the second gives

$$
\begin{aligned}
& g_{\alpha}^{i j} \phi_{i j k}=\left(g_{\alpha}^{i j} \psi_{i j}\right) * x_{k}+\hbar g_{\alpha}^{i j} \epsilon_{k i}^{m} \psi_{m j}+\frac{\hbar^{2}}{6} g_{\alpha}^{i j} \epsilon_{k i}^{m} \epsilon_{m j}^{p} x_{p}, \\
& g_{\alpha}^{j k} \phi_{i j k}=x_{i} *\left(g_{\alpha}^{j k} \psi_{j k}\right)+\hbar g_{\alpha}^{k j} \epsilon_{k i}^{m} \psi_{m j}+\frac{\hbar^{2}}{6} g_{\alpha}^{j k} \epsilon_{k i}^{m} \epsilon_{j m}^{p} x_{p} .
\end{aligned}
$$

Since $\phi$ is symmetric both right hand expressions must agree,

$$
\left(g_{\alpha}^{i j} \psi_{i j}\right) * x_{k}-x_{k} *\left(g_{\alpha}^{i j} \psi_{i j}\right)=2 \hbar g_{\alpha}^{i j} \epsilon_{i k}^{m} \psi_{m j} .
$$

The only equivariant tensors available for the 2-chain are the Killing form and, in the case of $\mathfrak{s l}_{n}$, a term linear in the generators, as above. Both satisfy this last condition, so of the two Equations (25) and (26) it is enough to examine the first. The problem of consistency of Equation (21) is reduced to the existence of $\phi_{i j k}$ that solves Equation (25).

A complete determination of the restricted cohomology is not required; it is enough to know the relations that define the orbit.

\subsection{CALCULATIONS FOR $\mathfrak{s l}_{n}$ : JOSEPH IDEAL AND HIGHEST WEIGHT MODULES}

6.3.1. Solving the constraints We use the notations and the commutation relations of $\mathfrak{s l}_{n}$ given in Section 5.4. We replace the general variables $x_{i}$ by the variables $U_{a}^{b}$ of $\mathfrak{s l}_{n}$, and Equations (22) and (24) take the form

$$
\frac{1}{2}\left(U_{a}^{b} * U_{c}^{d}+U_{c}^{d} * U_{a}^{b}\right)=U_{a}^{b} U_{c}^{d}+\psi_{a c}^{b d},
$$

and

$$
\begin{aligned}
\left(U_{a}^{b} U_{c}^{d}\right) * U_{e}^{f}= & U_{a}^{b} U_{c}^{d} U_{e}^{f}+\phi_{a c e}^{b d f}-\psi_{a c}^{b d} U_{e}^{f}-\psi\left(\psi_{a c}^{b d}, U_{e}^{f}\right)- \\
& -\frac{\hbar}{2}\left(\left\{U_{e}^{f}, U_{a}^{b}\right\} U_{c}^{d}+\left\{U_{e}^{f}, U_{c}^{d}\right\} U_{a}^{b}\right)- \\
& -\frac{\hbar^{2}}{12}\left(\left\{U_{a}^{b},\left\{U_{e}^{f}, U_{c}^{d}\right\}\right\}+\left\{U_{c}^{d},\left\{U_{e}^{f}, U_{a}^{b}\right\}\right\}\right) .
\end{aligned}
$$


Let us introduce a simpler notation to express the elements $u$ of $\mathfrak{g}^{\prime}$. We may write

$$
u=\sum_{a, b=1 \ldots n} A_{b}^{a} U_{a}^{b} .
$$

Set $U:=\left(U_{a}^{b}\right)_{a, b} \in \mathbf{M}_{n}\left(\mathfrak{g}^{\prime}\right)$ and $A:=\left(A_{a}^{b}\right)_{a, b} \in \mathbf{M}_{n} \mathbb{C}$. Then $u=(A U)$ is the trace of the matrix product $A U$. The coefficients $A_{b}^{a}$ are coordinates for $\mathfrak{g}, A$ ranges over the matrices of the adjoint representation of $\mathfrak{g}$. Equation (27) is linear in $U_{a}^{b}$ and $U_{c}^{d}$, so we may replace $U_{a}^{b}$ and $U_{c}^{d}$ by the linear combination $u:=(A U)$, and Equation (27) becomes

$$
u * u=u^{2}+\psi(u, u)
$$

Equivariance restricts $\psi$ :

$$
\psi(u, u)=k(A A U)+k^{\prime}(A A), \quad\left(k, k^{\prime}\right) \in \mathbb{C}^{2},
$$

where $(A A U)$ and $(A A)$ again stand for traces of products of matrices. Similarly,

$$
u * u * u=u^{3}+\phi(u, u, u)
$$

with

$$
\phi(u, u, u)=\phi_{1}(A A U) u+\phi_{2}(A A A U)+\phi_{3}(A A) u+\phi_{4}(A A A) .
$$

There are other invariants but their inclusion here is not allowed by the relations.

Let $\psi$ be given in the form Equation (29) and look at Equation (28) as an equation to determine $\phi$. Recall that the minimal orbit is defined by the relations

$$
U_{a}^{b} U_{c}^{d}=U_{a}^{d} U_{c}^{b}, \quad \forall a, b, c, d=1 \ldots n .
$$

PROPOSITION 6.6. Equation (28) is consistent with the relations $U_{a}^{b} U_{c}^{d}=U_{a}^{d} U_{c}^{b}$ if and only if the parameters $k$ and $k^{\prime}$ are related to each other as follows,

$$
4 k^{\prime}\left(1+\frac{1}{n}\right)=k^{2}\left(1+\frac{2}{n}\right)^{2}-\hbar^{2}
$$

Proof. See Appendix.

\subsubsection{Generators of the Joseph ideal}

PROPOSITION 6.7. The Joseph ideal for $\mathfrak{s l}_{n}$ is generated by the relations

$$
\begin{aligned}
U_{a}^{b} * U_{c}^{d}-U_{a}^{d} * U_{c}^{b}= & \frac{\hbar}{2}\left(\delta_{c}^{b} U_{a}^{d}-\delta_{a}^{d} U_{c}^{b}-\delta_{c}^{d} U_{a}^{b}+\delta_{a}^{b} U_{c}^{d}\right)+k^{\prime}\left(1+\frac{1}{n}\right)\left(\delta_{a}^{d} \delta_{c}^{b}-\delta_{a}^{b} \delta_{c}^{d}\right)+ \\
& +\frac{k}{2}\left(1+\frac{2}{n}\right)\left(\delta_{a}^{d} U_{c}^{b}+\delta_{c}^{b} U_{a}^{d}-\delta_{a}^{b} U_{c}^{d}-\delta_{c}^{d} U_{a}^{b}\right) .
\end{aligned}
$$


Proof. We find the relations of the deformed algebra by eliminating the original product from

$$
\begin{aligned}
U_{a}^{b} * U_{c}^{d}= & U_{a}^{b} U_{c}^{d}+\frac{\hbar}{2}\left(\delta_{c}^{b} U_{a}^{d}-\delta_{a}^{d} U_{c}^{b}\right)+k^{\prime}\left(\delta_{a}^{d} \delta_{c}^{b}-\frac{1}{n} \delta_{a}^{b} \delta_{c}^{d}\right)+ \\
& +\frac{k}{2}\left(\delta_{a}^{d} U_{c}^{b}+\delta_{c}^{b} U_{a}^{d}-\frac{2}{n}\left(\delta_{a}^{b} U_{c}^{d}+\delta_{c}^{d} U_{a}^{b}\right)\right)
\end{aligned}
$$

6.3.3. Highest weight module For a Lie algebra $\mathfrak{g}$, we choose a Cartan subalgebra $\mathfrak{h}$ consisting of diagonal matrices. Then a highest weight module of the deformed algebra is a module generated by a vector $v$ such that

$$
U_{a}^{b} * v=0, a<b=2 \ldots n, \quad U_{a}^{a} * v=\lambda_{a} v, a=1 \ldots n,
$$

with $\lambda \in \mathbb{C}^{n}$ and $\sum_{a=1}^{n} \lambda_{a}=0$. The vector $\lambda:=\left(\lambda_{1}, \ldots, \lambda_{n}\right)$ is called the weight of the vector $v$.

PROPOSITION 6.8. A highest weight module of the deformed algebra exists if and only if relation (31) holds. In that case there exists $m \in\{1, \ldots, n\}$ such that the highest weight $\lambda$ is given by

$$
\lambda_{1}=\lambda_{2}=\cdots=\lambda_{m-1}=-\frac{\hbar}{2}-\gamma, \quad \lambda_{m+1}=\cdots=\lambda_{n}=\frac{\hbar}{2}-\gamma
$$

where $\lambda_{m}$ is determined by $\sum_{i=1}^{n} \lambda_{i}=0$ and $\gamma=\frac{k}{2}\left(1+\frac{2}{n}\right)$.

Proof. The only relations that are changed by the deformation are those where $\{a, c\}$ intersects $\{b, d\}$, so we may limit ourselves to the case $b=a$. Also, if $a$ is equal to $c$ or to $d$ the relations are just the usual commutation relations. So take $c \neq a$ and $d \neq a$. Then

$$
U_{a}^{a} * U_{c}^{d}-U_{a}^{d} * U_{c}^{a}=\frac{\hbar}{2}\left(-\delta_{c}^{d} U_{a}^{a}+U_{c}^{d}\right)-\frac{k}{2}\left(1+\frac{2}{n}\right)\left(U_{c}^{d}+\delta_{c}^{d} U_{a}^{a}\right)-k^{\prime}\left(1+\frac{1}{n}\right) \delta_{c}^{d} .
$$

In particular, for $c=d$,

$$
U_{a}^{a} * U_{c}^{c}-U_{a}^{c} * U_{c}^{a}=\frac{\hbar}{2}\left(U_{c}^{c}-U_{a}^{a}\right)-\frac{k}{2}\left(1+\frac{2}{n}\right)\left(U_{c}^{c}+U_{a}^{a}\right)-k^{\prime}\left(1+\frac{1}{n}\right) .
$$

Applying this to a highest weight vector $v$ we obtain, for $a>c$,

$$
\lambda_{a} \lambda_{c}=\frac{\hbar}{2}\left(\lambda_{c}-\lambda_{a}\right)-\gamma\left(\lambda_{a}+\lambda_{c}\right)-\gamma^{\prime}, \quad \gamma:=\frac{k}{2}\left(1+\frac{2}{n}\right), \quad \gamma^{\prime}:=k^{\prime}\left(1+\frac{1}{n}\right) .
$$

Thus, for $a>c$,

$$
\left(\lambda_{c}+\frac{\hbar}{2}+\gamma\right) \lambda_{a}=\left(\frac{\hbar}{2}-\gamma\right) \lambda_{c}-\gamma^{\prime}
$$


Suppose $n>2$.

If $\gamma^{\prime} \neq \gamma^{2}-\left(\frac{\hbar}{2}\right)^{2}$, then $\lambda_{c} \neq-\frac{\hbar}{2}-\gamma$, hence

$$
\lambda_{a}=\frac{\left(\frac{\hbar}{2}-\gamma\right) \lambda_{c}-\gamma^{\prime}}{\lambda_{c}+\frac{\hbar}{2}+\gamma}, \quad a>c .
$$

Then taking $c=1$, we get $\lambda_{2}=\cdots=\lambda_{n}$, and taking $a=n$, we find $\lambda_{1}=\cdots=\lambda_{n-1}$. Since $\sum_{i=1}^{n} \lambda_{i}=0$, we have $\lambda_{i}=0$ for every $i$, which is not interesting.

We conclude that

$$
\gamma^{\prime}=\gamma^{2}-\left(\frac{\hbar}{2}\right)^{2},
$$

which is the same as Equation (31). Then there is an integer $m, 1 \leq m \leq n$ such that $\lambda_{m}$ differs from its neighbors, and the statement of the proposition follows immediately.

6.3.4. Abelian deformations When $\hbar=0$ the deformed algebra is commutative, so a representation is just a character, or a maximal ideal. The group acts on the maximal ideals, and among the maximal ideals there are upper triangular ones. This is possible only if either $n=2$ or if Equation (31) holds,

$$
4 k^{\prime}\left(1+\frac{1}{n}\right)=k^{2}\left(1+\frac{2}{n}\right)^{2} .
$$

In that case the deformed variety is the space of traceless matrices with all but one of the eigenvalues equal to $-\frac{k}{2}\left(1+\frac{2}{n}\right)$.

\subsection{CALCULATIONS FOR $\mathfrak{s o}_{n}$ : JOSEPH IDEAL AND HIGHEST WEIGHT MODULES}

6.4.1. Solving the constraints We use the notations and the commutation relations of $\mathfrak{s o}_{n}$ given in Sections 5.6.3. So, we now take for the general variables $x_{i}$ the variables $L_{a b}$ of $\mathfrak{s o}_{n}$, and Equation (22) takes the form

$$
\frac{1}{2}\left(L_{a b} * L_{c d}+L_{c d} * L_{a b}\right)=L_{a b} L_{c d}+\psi_{a b, c d} .
$$

Then Equation (24) now reads

$$
\begin{aligned}
\left(L_{a b} L_{c d}\right) * L_{e f}= & L_{a b} L_{c d} L_{e f}+\phi_{a b, c d, e f}-\psi_{a b, c d} L_{e f}- \\
& -\frac{\hbar}{2}\left(\left\{L_{e f}, L_{a b}\right\} L_{c d}+\left\{L_{e f}, L_{c d}\right\} L_{a b}\right)- \\
& -\frac{\hbar^{2}}{12}\left(\left\{L_{a b},\left\{L_{e f}, L_{c d}\right\}\right\}+\left\{L_{c d},\left\{L_{e f}, L_{a b}\right\}\right\}\right) .
\end{aligned}
$$

As for $\mathfrak{s l}_{n}$, a simpler notation is to write $u \in \mathfrak{g}^{\prime}$ as $u=(A L)=A^{a b} L_{a b}$. The coefficients $A^{a b}=-A^{b a}$ are coordinates for $\mathfrak{g}$. Then the two preceding equations become

$$
u * u=u^{2}+\psi(u, u),
$$


and

$$
u^{2} * v=u^{2} v+\phi(A, A, B)-\psi(A, A) v-\hbar[v, u]-\frac{\hbar^{2}}{6}\{u,\{v, u\}\} .
$$

Equivariance restricts $\psi$ and $\phi$,

$$
\psi_{A, A}=k(A A)
$$

and

$$
\phi(A A A)=\phi_{1}(A A A L)+\phi_{2}(A A) u
$$

where $(A A U)$ and $(A A)$ denote traces of products of matrices. In this context we take the form $\eta$ defined by $\eta_{a b}=0$ if $a \neq b$ and $\eta_{a a}=1$ for $a=1 \ldots n$, so that the two-forms become matrices without any fuss.

We fix the parameter $k$ and look at Equation (37) as an equation to determine the coefficients $\phi_{1}, \phi_{2}$. Then we have the following proposition.

PROPOSITION 6.9. Equation (37) is consistent with the relations that define the minimal orbit if and only if the parameter $k$ takes the value $\hbar \frac{n-4}{n-1}$. In this case the parameters $\phi_{1}$ and $\phi_{2}$ are fixed by Equation (37).

Proof. See Appendix.

\subsubsection{Generators of the Joseph ideal}

PROPOSITION 6.10. The Joseph ideal for $\mathfrak{s o}_{n}$ is generated by (the commutation relations and) the relations

$$
S\left(\eta^{b c} L_{a b} * L_{c d}\right)+\frac{\hbar^{2}}{2}(n-4) \eta_{a d}=0, \quad \sum_{\operatorname{cycl}(a b c)}\left(L_{a b} * L_{c d}-\hbar \eta_{a d} L_{b c}\right)=0
$$

These relations were derived by Binegar and Zierau [10], who also determined the highest weight module and the associated unitary representations of $\mathbf{S O}(p, q)$. We are interested, nevertheless, in deriving these results with the help of the starproduct. Equation (40) is a direct consequence of Equation (38), that reads in full

$$
L_{a b} * L_{c d}=L_{a b} L_{c d}+\frac{\hbar}{2}\left\{L_{a b}, L_{c d}\right\}+\frac{\ell}{2}\left(\eta_{a c} \delta_{b d}-\eta_{b c} \delta_{a d}\right) .
$$

6.4.3. Highest weight module for $\mathfrak{s o}_{n}$ Let $\left\{E_{a b} ; a, b=1 \ldots n\right\}$ denote the set of unit matrices of $\mathbf{M}_{n} \mathbb{C}$ as earlier, and take

$$
\eta:=\sum_{a=1}^{n} \delta_{a, a^{\prime}}, \text { with } a^{\prime}:=n+1-a
$$


A basis for $\mathfrak{s o}_{n}$ in the natural representation is the set $\left\{L_{a b} a<b \in\{1, \ldots, n\}\right\}$ of matrices

$$
L_{a b}:=\left(E_{a b}-E_{b a}\right) \eta=E_{a b^{\prime}}-E_{b a^{\prime}} .
$$

The Cartan subalgebra $\mathfrak{h}$ that we choose has for basis vectors

$$
H_{a}=L_{a a^{\prime}}-L_{a^{\prime} a}, \quad a=1 \ldots l .
$$

The set of positive root vectors is the collection $\left\{L_{a b} ; a+b \leq n, a<b\right\}$.

Our calculations are insensitive to the parity of $n$. Nevertheless we note the following facts:

$\triangleright$ When $n=2 l+1$, the simple root vectors are $L_{1, n-1}, L_{2, n-2}, \ldots, L_{l, l+1}$, and the associated simple roots are $\alpha_{i}=(0, \ldots, 0,1,-1,0, \ldots, 0), i=1 \ldots l-1$ with 1 in the $i$ 'th place and $\alpha_{l}=(0, \ldots, 0,1)$.

$\triangleright \quad$ When $n=2 l$, the simple root vectors are $L_{1, n-1}, L_{2, n-2}, \ldots, L_{l-1, l+1}, L_{l, l}$, and the associated simple roots are $\alpha_{i}=(0, \ldots, 0,1,-1,0, \ldots, 0), i=1 \ldots l-1$ with 1 in the $i^{\prime}$ th place and $\alpha_{l}=(0, \ldots, 0,1,1)$.

All this is in [12] (see also [34]).

Having fixed the root system and the Cartan subalgebra, we define a highest weight module as a module generated by a character $H_{a} \mapsto \lambda_{a} \in \mathbb{C}$ and a (highest weight) vector $v_{0}$ with the property

$$
\begin{aligned}
& \forall a+b \leq n, \quad L_{a b} v_{0}=0, \\
& \forall a=1 \ldots l, \quad H_{a} v_{0}=\hbar \lambda_{a} v_{0} .
\end{aligned}
$$

PROPOSITION 6.11. A highest weight module of the deformed algebra exists if and only if the parameter $\ell$ takes the value $\hbar^{2} \frac{n-4}{n-1}$. In that case, there is $k \in\{1, \ldots, l\}$ such that the highest weight $\lambda$ is given by

$$
\lambda_{1}=\cdots=\lambda_{k-1}=-1, \quad \lambda_{k}=k+1-\frac{n}{2}, \quad \lambda_{k+1}=\cdots=\lambda_{l}=0 .
$$

Proof. See Appendix. Joseph's choice is $k=l-1$.

In the formulation of Proposition 6.11 and of Equation (38a), in order to avoid confusion with the integer index $k$, we denoted by $\ell$ the complex parameter denoted by $k$ throughout this Section and the Appendix. In all other instances the context makes it clear which $k$ is meant.

When $n=2 l+1$, we have $\mathfrak{s o}_{n}=\mathfrak{s o}_{2 l+1}=B_{l}$, and

$$
\begin{aligned}
\lambda+\rho & =\omega_{1}+\cdots+\omega_{l-3}+\frac{1}{2}\left(\omega_{l-2}+\omega_{l-1}\right)+\omega_{l}= \\
& =\left(l-\frac{3}{2}, l-\frac{1}{2}, \ldots, \frac{5}{2}, \frac{3}{2}, 1, \frac{1}{2}\right), \\
\lambda & =\left(-1, \ldots,-1,-\frac{1}{2}, 0\right) .
\end{aligned}
$$


EXAMPLE 6.12. The case $n=5$ corresponds to the Bose singleton (a.k.a. the Rac). Another choice is $\lambda=\left(-1, \ldots,-1, \frac{1}{2}\right)$; in the case $n=5$ it is the Fermi singleton (a.k.a. the $\mathrm{Di}$ ). Both are unitarizable (after taking a quotient) representations of $\mathfrak{s o}(3,2)$. Binegar and Zierau take $k=1$.

When $n=2 l$, we have $\mathfrak{s o}_{n}=\mathfrak{s o}_{2 l}=D_{l}$, and Joseph again takes $k=l-1$ and

$$
\begin{aligned}
\lambda+\rho & =\omega_{1}+\cdots+\omega_{l-3}+\omega_{l-1}+\omega_{l}= \\
& =(l-3, l-2, \ldots, 1,1,0), \\
\lambda & =(-1,-1, \ldots,-1,0,0) .
\end{aligned}
$$

EXAMPLE 6.13. When $n=6$, this is the highest weight of the representation of the conformal group by a scalar massless field in 4 dimensions, the same representation that appears in Schrödinger's hydrogen atom. Binegar and Zierau take $k=1$.

\subsection{UNIFORM CALCULATIONS FOR THE EXCEPTIONAL SIMPLE LIE ALGEBRAS}

6.5.1. Minimal orbits of the exceptional simple Lie algebras Here we use some results of [63]. Let $\kappa$ denote the Killing form,

$$
\kappa\left(x_{i}, x_{j}\right)=\kappa_{i j}=-\operatorname{tr}\left(x_{i} x_{j}\right)=-\epsilon_{m i}{ }^{n} \epsilon_{n j}{ }^{m}
$$

and $\kappa^{i j}$ the matrix elements of the inverse matrix. The reduction of the symmetric part of the adjoint representation is governed by the operator $L: \mathfrak{g} \otimes \mathfrak{g} \rightarrow \mathfrak{g} \otimes \mathfrak{g}$ defined by

$$
L: x_{i} \otimes x_{j} \mapsto \kappa^{m n} \epsilon_{i m}{ }^{s} \epsilon_{j n}{ }^{t} x_{s} \otimes x_{t}=\kappa^{m n}\left[x_{i}, x_{m}\right] \otimes\left[x_{j}, x_{n}\right] .
$$

The symmetric part of the product $a d \otimes a d$ of the adjoint representation by itself decomposes into a direct sum of three representations, $I \oplus R_{2} \oplus R_{3}$. The last representation, that contains the extremal weights, is the minimal nilpotent orbit of $\mathfrak{g}$. The relations that define this orbit are the projections on the first two representations. The one-dimensional representation expresses the condition

$$
\kappa^{i j} x_{i} x_{j}=0 .
$$

The following table (see [63]) gives the eigenvalues $l_{i}=l_{1}, l_{2}, l_{3}$ associated with the representations $I, R_{2}, R_{3}$. In all cases, we have $l_{1}=1$, and $l_{2}+l_{3}=-\frac{1}{6}$.

\begin{tabular}{cccccc}
\hline $\mathfrak{g}$ & $G_{2}$ & $F_{4}$ & $E_{6}$ & $E_{7}$ & $E_{8}$ \\
\hline$l_{3}$ & $\frac{1}{4}$ & $\frac{1}{9}$ & $\frac{1}{12}$ & $\frac{1}{18}$ & $\frac{1}{30}$ \\
$D=\operatorname{dim} \mathfrak{g}$ & 27 & 52 & 78 & 133 & 248 \\
\hline
\end{tabular}


We turn to Equation (24). Actually, the exceptional simple Lie algebras are the easiest to deal with, and all five can be done uniformly. The immediate reason for this is the non existence of an irreducible invariant fourth order polynomial. The only equivariant symmetric tensors are (see Proposition 6.5)

$$
\psi_{i j}=k \kappa_{i j}, \quad \phi_{i j k}=\frac{k^{\prime}}{3} \sum_{\operatorname{cycl}(i j k)} \kappa_{i j} x_{k},
$$

where $k$ and $k^{\prime}$ are determined by the relations. So Equation (24) simplifies:

$$
\begin{gathered}
\left(x_{i} x_{j}\right) * x_{k}-x_{i} x_{j} x_{k}+\frac{\hbar}{2}\left(\left\{x_{k}, x_{i}\right\} x_{j}+(i \leftrightarrow j)\right)= \\
=\phi_{i j k}-\psi_{i j} x_{k}-\frac{\hbar^{2}}{12}\left(\left\{x_{i},\left\{x_{k}, x_{j}\right\}\right\}+(i \leftrightarrow j)\right) .
\end{gathered}
$$

\subsubsection{Main result}

THEOREM 6.14. Let $\mathfrak{g}$ be one of the five exceptional simple Lie algebras, and $\kappa$ the Killing form. There exists a unique, invariant star-product on the minimal nilpotent orbit, such that

$$
S\left(x_{i} * x_{j}\right)=x_{i} x_{j}+k \kappa_{i j}, \quad k \in \mathbb{C},
$$

for one and only one choice of $k, k^{\prime} \in \mathbb{C}$, namely $k=\frac{l_{2}-1}{D} \frac{\hbar^{2}}{6}+l_{2} \frac{\hbar^{2}}{12}, k^{\prime}=\frac{\hbar^{2}}{4}$.

Proof. It has already been pointed out that the only possible forms of the deformed products

$$
S\left(x_{i} * x_{j}\right)=x_{i} x_{j}+o(\hbar), \quad S\left(x_{i} * x_{j} * x_{k}\right)=x_{i} x_{j} x_{k}+o(\hbar)
$$

are

$$
S\left(x_{i} * x_{j}\right)=x_{i} x_{j}+\psi_{i j}, \quad S\left(x_{i} * x_{j} * x_{k}\right)=x_{i} x_{j} x_{k}+\phi_{i j k},
$$

with the equivariant maps $\psi, \phi$ as in Equation (40). The left hand side of Equation (41) satisfies the constraints. It remains to know if the right hand side does. Fix the index $k$ and define vectors $X, Y$ in $\mathfrak{g} \otimes \mathfrak{g}$ with components

$$
X_{i j}=\kappa_{i k} x_{j}+\kappa_{j k} x_{i}, \quad Y_{i j}=\kappa_{i j} x_{k} .
$$

Let $L_{s}$ denote the projection of the operator $L$ on the symmetric part of $\mathfrak{g} \otimes \mathfrak{g}$. Then the right hand side of Equation (41) is the vector

$$
\frac{k^{\prime}}{3}(X+Y)-k Y-\frac{\hbar^{2}}{12} L_{s} X \text {. }
$$

The operator $L_{s}$ satisfies the characteristic equation $\left(L_{s}-1\right)\left(L_{s}-l_{2}\right)\left(L_{s}-l_{3}\right)=0$, and since $L_{S} Y=Y$ there is a constant $c$ such that

$$
\left(L_{s}-l_{2}\right)\left(L_{s}-l_{3}\right) X=c Y \text {. }
$$


To determine the constant $c$, we contract this equation with $\kappa$ and find that

$$
c=\frac{2}{D}\left(1-l_{2}\right)\left(1-l_{3}\right) .
$$

With this, the vector (42) reduces to

$$
Z:=c^{-1}\left(\frac{k^{\prime}}{3}-k\right)\left(L_{s}-l_{2}\right)\left(L_{s}-l_{3}\right) X+\frac{k^{\prime}}{3} X-\frac{\hbar^{2}}{12} L_{s} X .
$$

Projecting on the trivial representation we get

$$
0=\frac{k^{\prime}}{3}(D+2)-k D-\frac{\hbar^{2}}{6}=0 .
$$

All the constraints are expressed by $\left(L_{s}-l_{3}\right) Z=0$, which yields the three conditions:

$$
\begin{aligned}
& \left(1-l_{3}\right) c^{-1}\left(\frac{k^{\prime}}{3}-k\right)-\frac{\hbar^{2}}{12}=0, \\
& \left(1-l_{3}\right) c^{-1}\left(\frac{k^{\prime}}{3}-k\right)\left(l_{2}+l_{3}\right)+\frac{k^{\prime}}{3}=l_{3} \frac{\hbar^{2}}{12}, \\
& \left(1-l_{3}\right) c^{-1}\left(\frac{k^{\prime}}{3}-k\right) l_{2} l_{3}=l_{3} \frac{k^{\prime}}{3} .
\end{aligned}
$$

All four Equations (43)-(44) agree on the unique values of $k$ and $k^{\prime}$

$$
k^{\prime}=l_{2} \frac{\hbar^{2}}{4}, \quad k-\frac{k^{\prime}}{3}=\frac{l_{2}-1}{D} \frac{\hbar^{2}}{6} .
$$

The proposition is proved.

6.5.3. Generators of the Joseph ideal The Joseph ideal is of course generated by the relations

$$
x_{i} * x_{j}-x_{j} * x_{i}=\hbar\left\{x_{i}, x_{j}\right\},
$$

and

$$
\left(L_{s}-l_{3}\right)\left(x_{i} * x_{j}-k \kappa_{i j}\right)=0 .
$$

Open Access This article is distributed under the terms of the Creative Commons Attribution Noncommercial License which permits any noncommercial use, distribution, and reproduction in any medium, provided the original author(s) and source are credited. 


\section{Appendix}

Proof of Proposition 6.6. The lack of symmetry in Equation (28) forces us to introduce a second variable, denoted by $v=(B U)$. Then the equation can be written as

$$
u^{2} * v=u^{2} v+\phi(u, u, v)-\psi(u, u) v-\psi(\psi(u, u), v)-\hbar\{v, u\} u-\frac{\hbar^{2}}{6}\{u,\{v, u\}\},
$$

with $\phi$ given by Equation (30),

$$
\begin{aligned}
\phi(u, u, v)= & \frac{\phi_{1}}{3}((A A U) v+2(A B U) u)+\frac{\phi_{2}}{3}((A A B U)+(A B A U)+(B A A U))+ \\
& +\frac{\phi_{3}}{3}((A A) v+2(A B) u)+\phi_{4}(A A B) .
\end{aligned}
$$

Exchange of the upper indices on the two A's is a type of Fierz transformation. The effect of this exchange is the following:

$$
\begin{aligned}
(A B A U) & \mapsto(A B) u-\frac{1}{n}(A A B U)-\frac{1}{n}(A A U B)+\frac{1}{n^{2}}(A A) v, \\
(A A B U) & \mapsto-\frac{2}{n}(A A B U)+\frac{1}{n^{2}}(A A) v, \\
(A B U) u & \mapsto(A B U) u-\frac{1}{n}(A A U) v, \\
(B A U) u & \mapsto(B A U) u-\frac{1}{n}(A A U) v, \\
(A A U) & \mapsto-\frac{2}{n}(A A U), \\
(A A B) & \mapsto-\frac{2}{n}(A A B), \\
(A B) u & \mapsto(A U A B), \\
(A A) & \mapsto-\frac{1}{n}(A A) .
\end{aligned}
$$

For example, consider $(A B) u=\left(\sum_{a, b} A_{a}^{b} B_{b}^{a}\right)\left(\sum_{c, d} A_{c}^{d} U_{d}^{c}\right)$. After the exchange of the upper indices on the two A's, this expression becomes $\sum_{a, b, c, d} A_{a}^{d} U_{d}^{c} A_{c}^{b} B_{b}^{a}=$ $(A U A B)$.

In the same way,

$$
\begin{aligned}
\phi(u, u, v) \mapsto & \frac{\phi_{1}}{3}\left(-\frac{2}{n}(A A U) v+2(A B U) u-\frac{2}{n}(A A U) v\right)+ \\
& +\frac{\phi_{2}}{3}\left(-\frac{3}{n}(A A B U+A A U B)+\frac{3}{n^{2}}(A A) v+(A B) u\right)+ \\
& +\frac{\phi_{3}}{3}\left(-\frac{1}{n}(A A) v+2(A B A U)\right)+\phi_{4}\left(\frac{-2}{n}(A A B)\right) .
\end{aligned}
$$


Furthermore, we have

$$
\begin{aligned}
-\psi(u, u) v= & -k(A A U) v-k^{\prime}(A A) v \mapsto \frac{2 k}{n}(A A U) v+\frac{k^{\prime}}{n}(A A) v, \\
-\psi(\psi(u, u), v)= & -\frac{k^{2}}{2}\left((A A B U+A A U B)-\frac{2}{n}(A A) v\right)-k k^{\prime}(A A B) \mapsto \\
\mapsto & \frac{k^{2}}{n}\left((A A B U+A A U B)-\frac{2}{n}(A A) v\right)- \\
-\frac{\hbar^{2}}{6}\{u,\{v, u\}\}= & -\frac{k^{2}}{n}(A A) v+\frac{k k^{\prime}}{n}(A A B), \\
\mapsto & \frac{\hbar^{2}}{3}\left(-(A B) u+\frac{1}{n}(A A B U+A A U B)-\frac{1}{n^{2}}(A A) v\right)- \\
& -\frac{\hbar^{2}}{6}\left(-\frac{2}{n}(A A B U+A A U B)+\frac{2}{n^{2}}(A A) v\right) .
\end{aligned}
$$

Applying the constraint $U_{a}^{b} U_{c}^{d}=U_{a}^{d} U_{c}^{d}$ to Equation (28), we get the following equations

$$
\begin{aligned}
& \frac{1}{3} \phi_{1}\left(1+\frac{2}{n}\right)-k\left(1+\frac{2}{n}\right)=0 \\
& 2 \phi_{2}\left(1+\frac{3}{n}\right)-3 k^{2}\left(1+\frac{2}{n}\right)+\hbar^{2}=0 \\
& \phi_{2}-2 \phi_{3}-\hbar^{2}=0 \\
& \phi_{3}\left(1+\frac{1}{n}\right)-3 k^{\prime}\left(1+\frac{1}{n}\right)-\frac{3}{n^{2}} \phi_{2}+\frac{3}{n} k^{2}\left(1+\frac{2}{n}\right)=0 \\
& \phi_{4}\left(1+\frac{2}{n}\right)-k k^{\prime}\left(1+\frac{2}{n}\right)=0
\end{aligned}
$$

We eliminate the parameters $\phi_{i}$ 's and we find the relation

$$
4 k^{\prime}\left(1+\frac{1}{n}\right)=k^{2}\left(1+\frac{2}{n}\right)^{2}-\hbar^{2} .
$$

The proposition is proved.

Proof of Proposition 6.9. Recall that the relations that define the minimal nilpotent orbit are

$$
\sum_{\operatorname{cycl}(a b c)} L_{a b} L_{c d}=0, \quad \eta^{b c} L_{a b} L_{c d}=0
$$


By applying the first relation (contraction on $b, c$ ) to Equation (37), we get

$$
\begin{aligned}
0= & \eta^{b c} \phi_{a b, c d, e f}-\frac{k}{2}(n-1) \eta_{a d} L_{e f}-\frac{\hbar^{2}}{12}\left(( n - 4 ) \left(\eta_{f d} L_{a e}+(a \leftrightarrow d)-\right.\right. \\
& \left.-(e \leftrightarrow f)-(a \leftrightarrow d, e \leftrightarrow f))-4 \eta_{a d} L_{e f}\right),
\end{aligned}
$$

and

$$
\begin{aligned}
\eta^{b c} \phi_{a b, c d, e f}= & \left(\frac{\phi_{1}}{24}(n-1)-\frac{\phi_{2}}{6}\right)\left(\eta_{f d} L_{a e}+(a \leftrightarrow d)-(e \leftrightarrow f)-(a \leftrightarrow d, e \leftrightarrow f)\right)+ \\
& +\left(-\frac{\phi_{1}}{6}+\frac{\phi_{2}}{6}(n-1)\right) \eta_{a d} L_{e f} .
\end{aligned}
$$

The second relation (a kind of Fierz transformation) gives

$$
0=\sum_{\mathrm{cycl}} \phi_{a b, c d, e f}+\frac{\hbar^{2}}{3} \eta_{f a}\left(\eta_{d e} L_{b c}+\eta_{e c} L_{d b}\right)-(e \leftrightarrow f)
$$

and

$$
\sum_{\mathrm{cycl}} \phi_{a b, c d, e f}=\left(\frac{\phi_{1}}{12}+\frac{\phi_{2}}{6}\right) \sum_{\mathrm{cycl}} \eta_{f a}\left(\eta_{d e} L_{c b}+\eta_{c e} L_{b d}\right)-(e \leftrightarrow f) .
$$

Hence the equations:

$$
\begin{aligned}
& \frac{\phi_{1}}{24}(n-1)-\frac{\phi_{2}}{6}-\frac{\hbar^{2}}{12}(n-4)=0, \\
& -\frac{\phi_{1}}{6}+\frac{\phi_{2}}{6}(n-1)-\frac{k}{2}(n-1)+\frac{\hbar^{2}}{3}=0, \\
& \frac{\phi_{1}}{12}+\frac{\phi_{2}}{6}+\frac{\hbar^{2}}{3}=0 .
\end{aligned}
$$

Finally, we obtain

$$
\begin{aligned}
\phi_{1}(n+1) & =2 \hbar^{2}(n-8) \\
\phi_{2}(1+n) & =-3 \hbar^{2}(n-2) \\
k(n-1) & =\hbar^{2}(n-4) .
\end{aligned}
$$

The proposition is proved.

Proof of Proposition 6.11. All Cartan subalgebras are isomorphic and any two systems of simple roots are related by a transformation of the Weyl group. The ideal determines only the infinitesimal character $\chi(\lambda):=\rho+\lambda$, where $\rho$ is half the sum of the positive roots and $\lambda$ is the highest weight, up to a Weyl reflection. It does not distinguish between weights that are related by a Weyl transformation of the infinitesimal character. In the case of $B_{l}=\mathfrak{s o}_{2 l+1}$ the formula is $\rho=\left(l-\frac{1}{2}\right.$, $\left.l-\frac{3}{2}, \ldots, \frac{1}{2}\right)$. 
The problem is to determine the possible values of the infinitesimal character. We begin with the relation

$$
\sum_{b} L_{d b} * L_{b^{\prime} a}-\frac{\hbar}{2}(n-2) L_{d a}+\frac{k}{2}(n-1) \eta_{d a}=0,
$$

in the case $a+d=n+1, a<d$. Applied to the highest vector $v_{0}$, it gives

$$
\left(\sum_{b \leq a} L_{d b} * L_{b^{\prime} a}+\frac{\hbar}{2}(n-2) \lambda_{a}+\frac{k}{2}(n-1)\right) v_{0}=0 .
$$

If $b>a$, then $b^{\prime}<a^{\prime}$ and $a+b^{\prime}<n+1$, so $L_{b^{\prime} a} v_{0}=0$. Thus

$$
\left(\sum_{b<a}\left\{L_{d b}, L_{b^{\prime} a}\right\}+\lambda_{a} 2+\frac{\hbar}{2}(n-2) \lambda_{a}+\frac{k}{2}(n-1)\right) v_{0}=0 .
$$

Finally, as the bracket is equal to $\hbar^{2}\left(\lambda_{b}-\lambda_{a}\right)$, for $b=1 \ldots a-1$, we have

$$
(1-a) \lambda_{a}+\left(\lambda_{1}+\cdots+\lambda_{a-1}\right)+\lambda_{a}^{2}+\frac{1}{2}(n-2) \lambda_{a}+\frac{1}{2}(n-4) .
$$

In particular

$$
\begin{aligned}
& \lambda_{1}^{2}+\frac{1}{2}(n-2) \lambda_{1}+\frac{1}{2}(n-4)=0, \\
& \left(\lambda_{1}+1\right)\left(\lambda_{1}+\frac{n-4}{2}\right)=0 .
\end{aligned}
$$

Thus

$$
(1-a) \lambda_{a}+\left(\lambda_{1}+\cdots+\lambda_{a-1}\right)+\lambda_{a}^{2}-\lambda_{1}^{2}+\frac{n-2}{2}\left(\lambda_{a}-\lambda_{1}\right)
$$

and

$$
\left(\lambda_{a}-\lambda_{a-1}\right)\left(\lambda_{a}+\lambda_{a-1}+\frac{1}{2}(n-a)\right)=0 .
$$

We return to the relation

$$
\sum_{b} L_{d b} * L_{b^{\prime} a}-\frac{\hbar}{2}(n-2) L_{d a}+\frac{k}{2}(n-1) \eta_{d a}=0,
$$

now in the case $a+d=n+2,2 \leq a<d$. Applying this relation to the highest weight vector $v_{0}$, we have

$$
\begin{aligned}
& \left(\sum_{b \leq a} L_{d b} * L_{b^{\prime} a}-\frac{\hbar}{2}(n-2) L_{d a}\right) v_{0}=0, \\
& \left(\sum_{b \leq a-2} L_{d b} * L_{b^{\prime} a}+L_{d a} * L_{a^{\prime} a}+L_{d d^{\prime}} L_{d a}-\frac{\hbar}{2}(n-2) L_{d a}\right) v_{0}=0 .
\end{aligned}
$$


The first term can be replaced by the bracket $\sum_{a \leq a-2}\left[L_{d b}, L_{b^{\prime} a}\right]=\hbar \sum_{b \leq a-2} L_{d a}$. So, for $a=2 \ldots l$, we have the relation

$$
\left((a-1) L_{d a}-\lambda_{a} L_{d a}-\lambda_{a-1} L_{d a}-\frac{1}{2}(n-2) L_{d a}\right) v_{0}=0 .
$$

Hence

$$
\left(\lambda_{a}+\lambda_{a-1}+\frac{1}{2}(n-2 a)\right) L_{d a} v_{0}=0, \forall a=2 \ldots l .
$$

Note that $\left\{L_{d a}, L_{a^{\prime} d^{\prime}}\right\}=\hbar^{2}\left(\lambda_{a}-\lambda_{a-1}\right) v_{0}$. The information contained in this last result is therefore precisely the same as in Equation (45), with $\lambda_{2}=\cdots=\lambda_{n}=0$. Then $L_{a b} v_{0}=0, a, b>1$.

Next, the other relation,

$$
\sum_{\operatorname{cycl}(b c d)}\left(L_{a b}-\hbar \eta_{a b}\right) * L_{c d}=0,
$$

applied to $v_{0}$ in the case $a+b=c+d=n+1, a<b, c<d, a \neq c$, gives

$$
\left(\left(\lambda_{a}-1\right) \lambda_{c}+L_{a c} * L_{d b}+L_{a d} * L_{b c}\right) v_{0}=0 .
$$

By evaluating this in two cases, $a>b, a<c$, we find that $\lambda_{<}$is -1 or $\lambda_{>}$is 0 . This completes the proof.

\section{References}

1. Abellanas, L., Martinez-Alonso, L.: Quantization from the algebraic viewpoint. J. Math. Phys. 17(8), 1363-1365 (1976)

2. Agarwal, G.S., Wolf, E.: Calculus for functions of noncommuting operators and general phase-space methods in quantum mechanics. I. Mapping theorems and ordering of functions of noncommuting operators. Phys. Rev. D2, 2161-2186 (1970)

3. Barr, M.: Cohomology of Commutative Algebras. Dissertation, U. Penn. (1962)

4. Barr, M.: Harrison homology, Hochschild homology and triples. J. Algebra 8, 314-323 (1968)

5. Bayen, F., Flato, M., Fronsdal, C., Lichnerowicz, A., Sternheimer, D.: Deformation theory and quantization. Ann. Phys. 111, 61-110, 111-151 (1978)

6. Bayen, F., Fronsdal, C.: Quantization on the sphere. J. Math. Phys. 22, 1345-1349 (1981)

7. Beilinson, A., Ginsburg, V., Schechtman, V.: Koszul duality. J. Geom. Phys. 5, 317-350 (1988)

8. Berezin, F.A.: General concept of quantization. Commun. Math. Phys. 40, 153-174 (1975)

9. Bezrukavnikov, R.: Koszul property and Frobenius splitting of Schubert varieties. arXiv:alg-geom/9502021v1

10. Binegar, B., Zierau, R.: Unitarization of a singular representation of $\mathbf{S O}(p, q)$. Commun. Math. Phys. 138, 245-258 (1991)

11. Borho, W., Brylinski, J.: Differential operators on homogeneous spaces I: Irreducibility of the associated variety. Inv. Math. 69, 437-476 (1982) 
12. Bourbaki: Groupes et algèbres de Lie. Masson, Paris (1981)

13. Braverman, A., Joseph, A.: The minimal realization from deformation Theory. J. Algebra 205, 13-16 (1998)

14. Brylinski, R.: Geometric quantization of real minimal nilpotent orbits, symplectic geometry. Diff. Geom. Appl. 9(1-2), 5-58 (1998). arXiv:math/9811033v1[math.SG]

15. Cahen, M., Gutt, S., Rawnsley, J.: On tangential star products for the coadjoint Poisson structure. Commun. Math. Phys. 180, 99-108 (1996)

16. Cattaneo, A., Keller, B., Torossian, C., Bruguières, A.: Déformation, quantification, théorie de Lie. Panoramas et Synthèses, vol. 20. Société Mathématique de France, Paris (2005)

17. Collingwood, D.H., McGovern, W.M.: Nilpotent Orbits in Semisimple Lie Algebras. Van Nostrand Reinhold, New York (1993)

18. Dixmier, J.: Algèbres Enveloppantes. Gauthier-Villars Editeur, Paris (1974)

19. Duflo, M.: Sur la classification des idéaux primitifs dans l'algèbre enveloppante d'une algèbre de Lie semi-simple. Ann. Math. 105, 107-120 (1977)

20. De Wilde, M., Lecomte, P.B.A.: Existence of star-products and of formal deformations of the Poisson algebra of arbitrary symplectic manifolds. Lett. Math. Phys. 7, 487-496 (1983)

21. Fedosov, B.V.: A simple geometrical construction of deformation quantization. J. Diff. Geom. 40, 213-238 (1994)

22. Fioresi, R., Lledo, M.A.: On the deformation quantization of coadjoint orbits of semisimple Lie groups. Pac. J. Math. 198(2), 411-436 (2001)

23. Fioresi, R., Lledo, M.A.: A comparison between star products on regular orbits of compact Lie groups. J. Phys. A 35, 5687-5700 (2002). arXiv:math/0106129v3 [math.QA]

24. Fioresi, R., Levrero, A., Lledo, M.A.: Algebraic and differential star products on regular orbits of compact Lie groups. Pac. J. Math. 206, 321-337 (2002). arXiv:math/ 0011172v2 [math.QA]

25. Fioresi, R., Lledo, M.A., Varadarajan, V.S.: On the deformation quantization of affine algebraic varieties. Int. J. Math. 16, 419-436 (2005). arXiv:math/0406196v1 [math.QA]

26. Flato, M., Lichnerowicz, A., Sternheimer, D.: Déformations 1-différentiables d'algèbres de Lie attachées à une variété symplectique ou de contact. C. R. Acad. Sci. Paris Ser. A 279, 877-881 (1974)

27. Flato, M., Lichnerowicz, A., Sternheimer, D.: Deformations of Poisson brackets, Dirac brackets and applications. J. Math. Phys. 17, 1754-1762 (1976)

28. Fleury, P.J.: Splittings of Hochschild's complex for commutative algebras. Proc. AMS 30, 323-405 (1971)

29. Fronsdal, C.: Some ideas about quantization. Rep. Math. Phys. 15, 111-145 (1978)

30. Frønsdal, C.: Harrison cohomology and Abelian deformation quantization on algebraic varieties. Deformation quantization (Strasbourg, 2001). In: IRMA Lect. Math. Theor. Phys., vol. 1, pp. 149-161. de Gruyter, Berlin (2002). arXiv:hep-th/0109001v3

31. Fronsdal, C.: Abelian deformations. In: Proceedings of the IX'th International Conference on Symmetry Methods in Physics, Yerevan, July 2001

32. Fronsdal, C., Galindo, A.: The ideals of free differential algebras. J. Algebra 222, 708-746 (1999). arXiv:math/9806069v2 [math.QA]

33. Frønsdal, C., Kontsevich, M.: Quantization on curves. Lett. Math. Phys. 79, 109-129 (2007). arXiv:math-ph/0507021v2

34. Fulton, W., Harris, J.: Representation Theory. Springer, New York (1991)

35. Gan, Wee Teck., Savin, G.: Uniqueness of the Joseph ideal. Math. Res. Lett. 11, 589597 (2004) 
36. Garsia, A.M.: Combinatorics of the free Lie algebra and the symmetric group. In: Analysis, et cetera, Research Papers Published in Honor of Jürgen Moser's 60'th Birthday, pp. 209-362. Academic Press, New York (1990)

37. Gerstenhaber, M.: The cohomology structure of an associative ring. Ann. Math. (2) 78, 267-288 (1963)

38. Gerstenhaber, M.: On the deformations of rings and algebras. Ann. Math. 79, 59-103 (1964)

39. Gerstenhaber, M.: Developments from Barr's thesis, presented at the celebration of the 60'th birthday of Michael Barr (30 June 1998). J. Pure Appl. Algebra 143, 205-220 (1999)

40. Gerstenhaber, M., Schack, S.D.: A Hodge-type decomposition for commutative algebra cohomology. J. Pure Appl. Algebra 48, 229-247 (1987)

41. Guieu, L., Roger, C.: Avec un appendice de Vlad Sergiescu. L'Algèbre et le Groupe de Virasoro: aspects géométriques et algébriques, généralisations, Publication du Centre de Recherches Mathématiques de Montréal, Monographies, notes de cours et Actes de conférences, PM28 (2007)

42. Gukov, S., Witten, E.: Branes and Quantization. arXiv:0809.0305v2[hep-th]

43. Gutt, S.: An explicit star-product on the cotangent bundle of a Lie group. Lett. Math. Phys. 7, 249-258 (1983)

44. Halbout, G.: Oudom J.-M., Tang X., Deformations of Linear Poisson Orbifolds. arXiv: 0807.0027v1[math.QA]

45. Halbout, G., Tang, X.: Noncommutative Poisson Structures on Orbifolds. arXiv: math/0606436v2[math.QA]

46. Harrison, D.K.: Commutative algebras and cohomology. Trans. Am. Math. Soc. 104, 191-204 (1962)

47. Hochschild, G., Kostant, B., Rosenberg, A.: Differential forms on regular affine algebras. Trans. Am. Math. Soc. 102, 383-408 (1962)

48. Humphreys, J.E.: Introduction to Lie Algebras and Representation Theory. Springer, New York (1972)

49. Joseph, A.: Minimal realizations and spectrum generating algebras. Commun. Math. Phys. 36, 325-338 (1974)

50. Joseph, A.: The minimal orbit in a simple Lie algebra and its associated maximal ideal. Ann. Sci. Ecol. Norm. Sup. 9, 1-30 (1976)

51. Joseph, A.: Dixmier's problem for Verma and principal series submodules. J. Lond. Math. Soc. 20, 193-204 (1979)

52. Joseph, A.: On the associated variety of a primitive ideal. J. Algebra 93, 509-523 (1985)

53. Kirillov, A.A.: Eléments de la Théorie des Représentations. Editions Mir, Moscou (1974)

54. Kirillov, A.A.: Lectures on the Orbit Method, Graduates Studies in Mathematics, vol. 64, American Mathematical Society, Providence, Rhode Island (2004)

55. Kontsevich, M.: Operads and motives in deformation quantization. Lett. Math. Phys. 48, 35-72 (1999). arXiv:math/9904055v1 [math.QA]

56. Kontsevich M.: Deformation Quantization of algebraic varieties. In: Euro Conference Moshé Flato 2000, Part III (Dijon). Lett. Math. Phys. 56, 271-294 (2001). arXiv:math/0106006v1 [math.AG]

57. Kontsevich, M.: Deformation quantization of Poisson manifolds. Lett. Math. Phys. 66, 157-216 (2003). arXiv:q-alg/9709040v1

58. Kostant, B.: Lie group representations on polynomial rings. Am. J. Math. 85, 327-404 (1963) 
59. Kostant, B.: Quantization and unitary representations. Lectures in Modern Analysis and Applications III, Lecture Notes in Mathematics, vol. 170, pp. 87-208. Springer, Berlin (1970)

60. Lledo, M.A.: Deformation quantization of non regular orbits of compact Lie groups. Lett. Math. Phys. 58, 57-67 (2001). arXiv:math/0105191v3 [math.QA]

61. Loday, J.-L.: Opérations sur l'homologie cyclique des algèbres commutatives. Invent. Math. 96, 205-230 (1989)

62. Moyal, J.E.: Quantum mechanics as a statistical theory. Proc. Camb. Phil. Soc. 45, 99124 (1979)

63. Macfarlane, J.A., Pfeiffer, H.: Development of a unified tensor calculus for exceptional lie algebras. Int. J. Mod. Phys. A 19, 287-316 (2004). arXiv:math-ph/0212047v1

64. Marsden, J.E., Ratiu, T.S.: Introduction to Mechanics and Symmetry, 2nd edn. Springer, Heidelberg (1999)

65. Postnikov, M.: Leçons de Géométrie, Groupes et Algèbres de Lie. Editions Mir, Moscou (1982)

66. Souriau, J.M.: Structures des Systèmes Dynamiques. Dunod, Paris (1970)

67. Tamarkin, D.E.: Another proof of M. Kontsevich formality theorem for $\mathbb{R}^{n}$. math.QA/ $9803025 \mathrm{v} 4$

68. Vey, J.: Déformation du crochet de Poisson sur une variété symplectique. Comment. Math. Helv. 50, 421-454 (1975)

69. Weinstein, A.: Deformation quantization, Séminaire Bourbaki. Astérisque, vol.1993/ 1994, No. 227, Exp. No. 789, 5, 389-409 (1995)

70. Weyl, H.: Theory of Groups and Quantum Mechanics. Dover, New York (1931)

71. Wigner, E.P.: Quantum corrections for thermodynamic equilibrium. Phys. Rev. 40, 749759 (1932) 